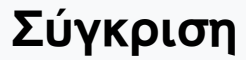

Tóp. 22 (2012)

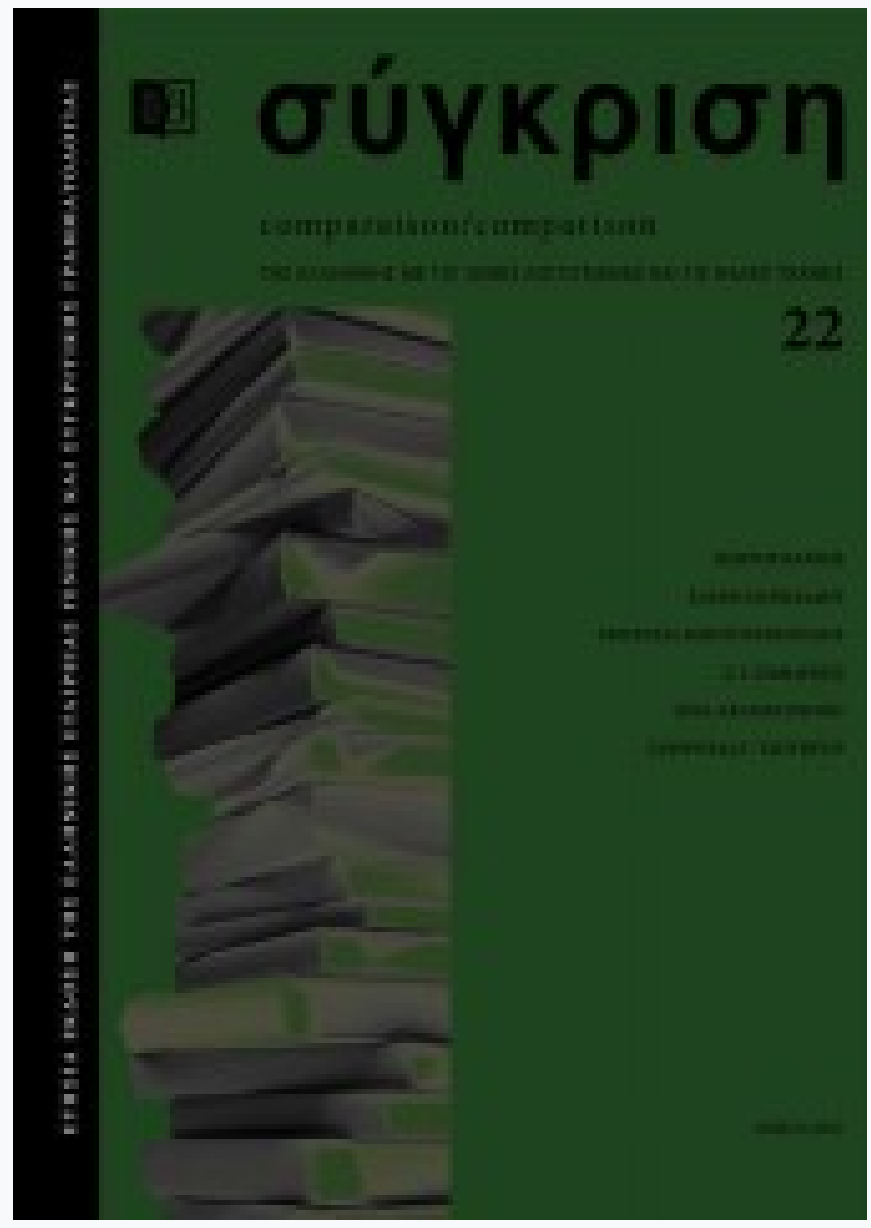

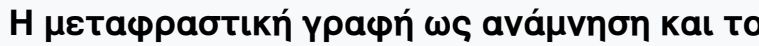

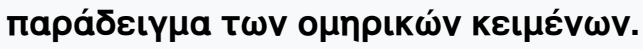

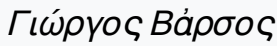

doi: $10.12681 /$ comparison.28

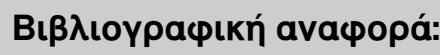

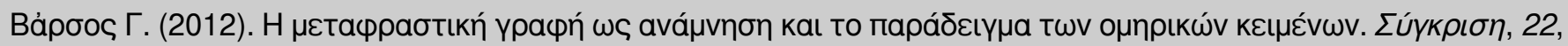
7-20. https://doi.org/10.12681/comparison.28 


\title{
TIRPIOE BAPEOE
}

\author{
$\mathrm{H} \mu \varepsilon \tau \alpha \varphi \rho \alpha \sigma \tau \iota x \dot{n}^{\prime} \gamma \rho \alpha \varphi \dot{\eta} \omega \varsigma \alpha \nu \alpha \dot{\mu \nu \eta \sigma \eta} x \alpha \iota$

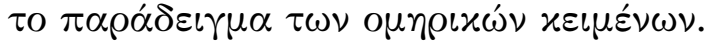

$\gamma^{\prime}$

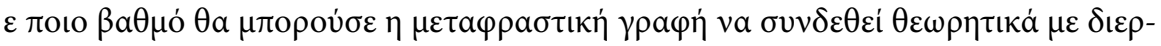

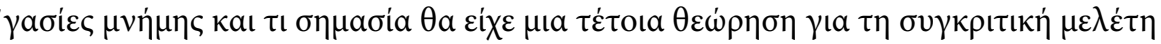

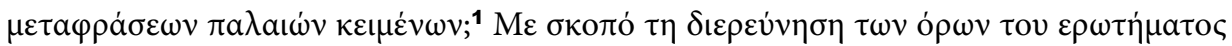

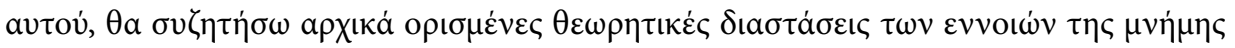

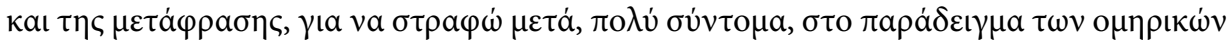

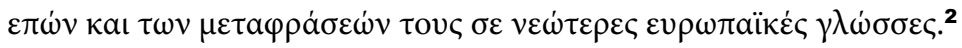

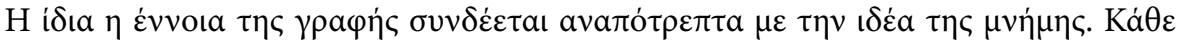

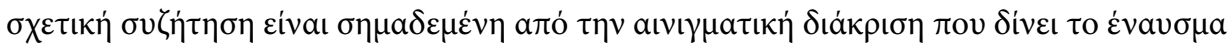

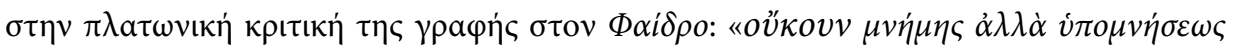

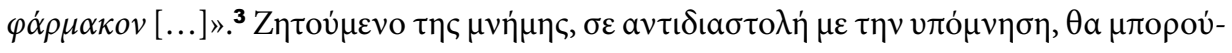

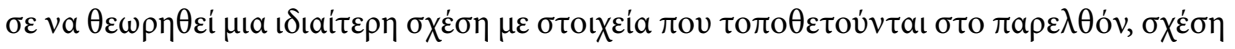

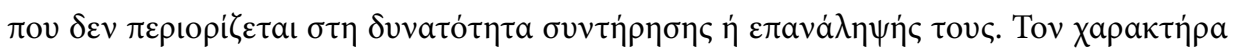

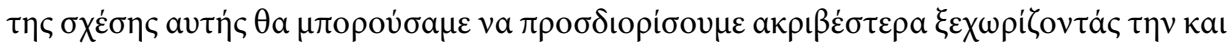

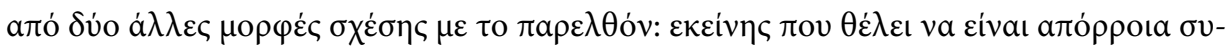

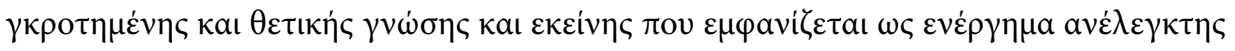

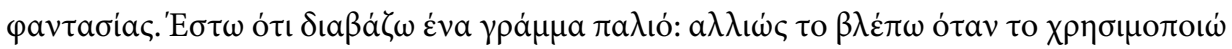

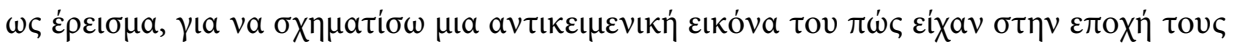
o

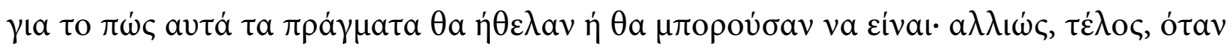

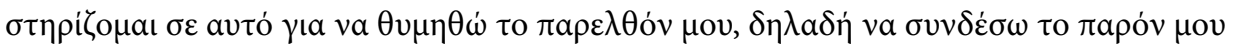

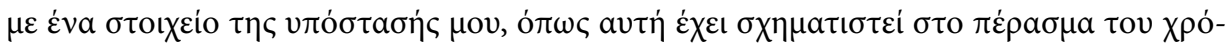

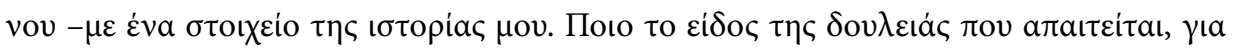

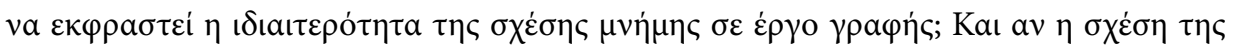

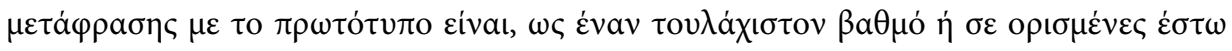

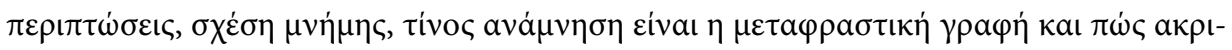

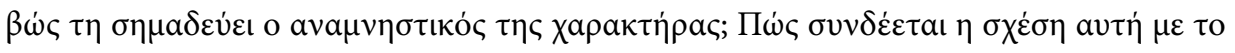

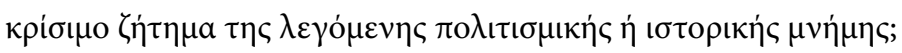

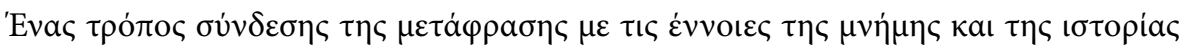

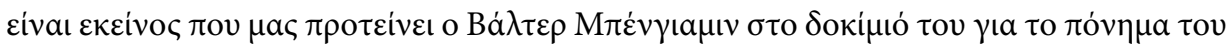

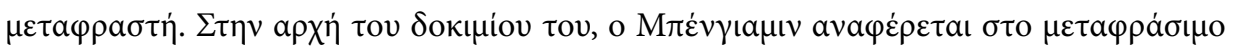




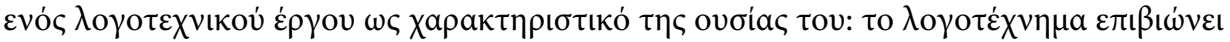

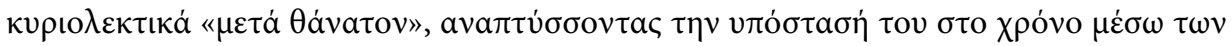

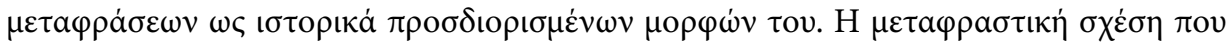

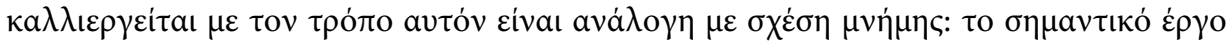

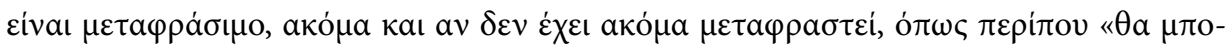

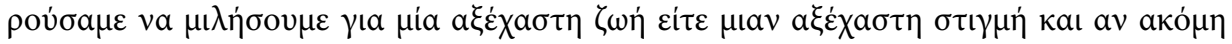

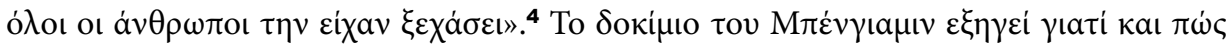

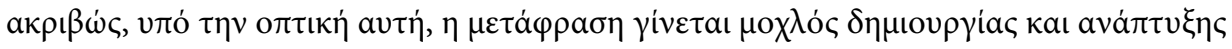
каi

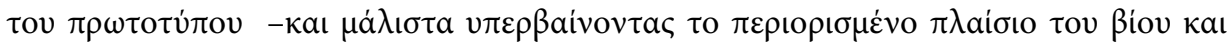

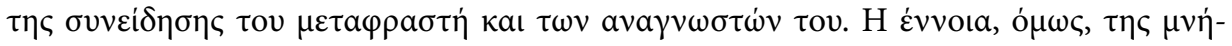

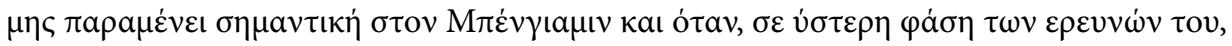

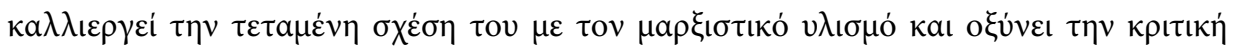

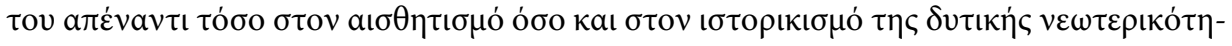

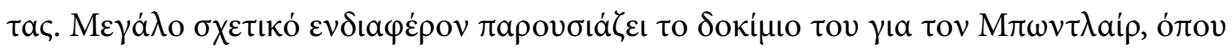

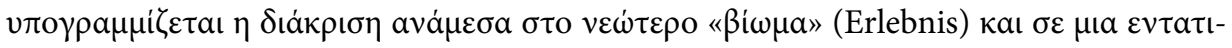

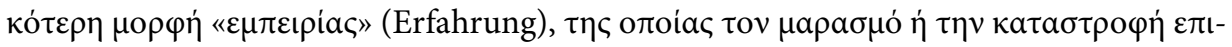

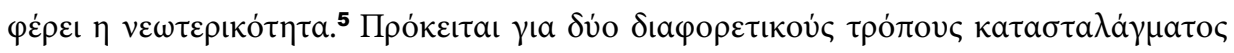

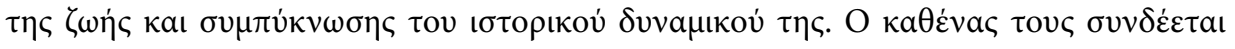

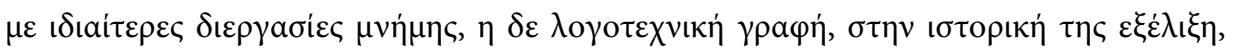

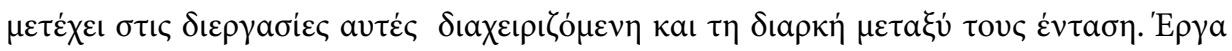

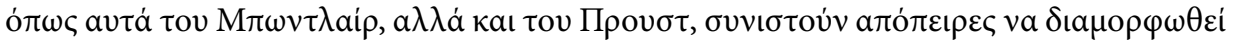

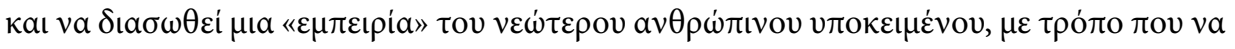

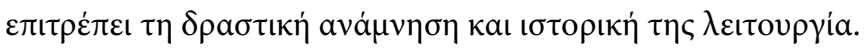

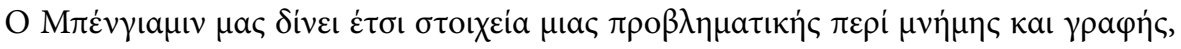

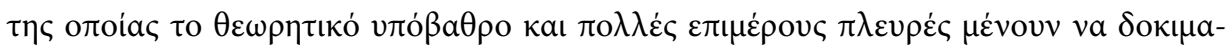

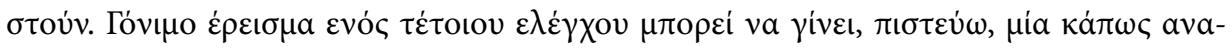

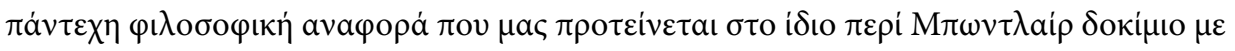

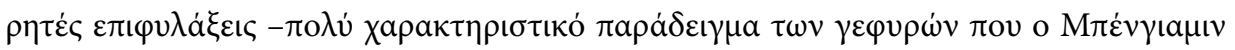

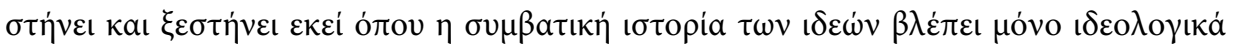

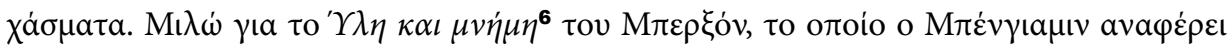

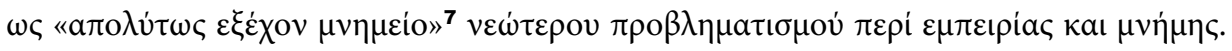

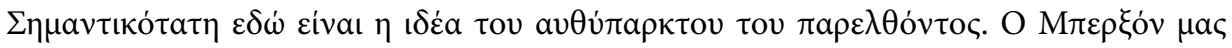

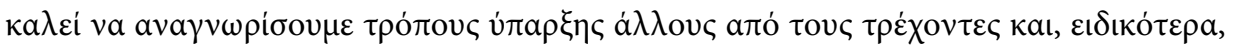

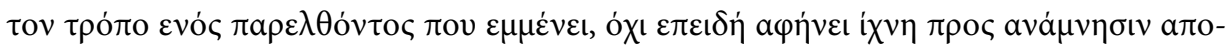

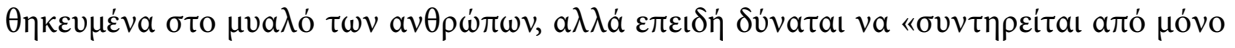

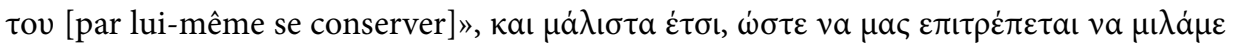




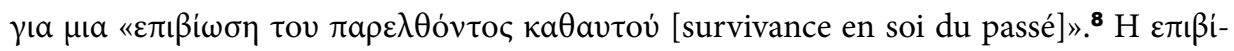

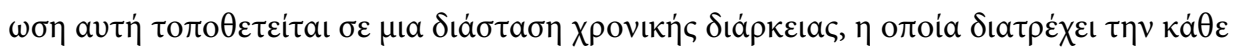

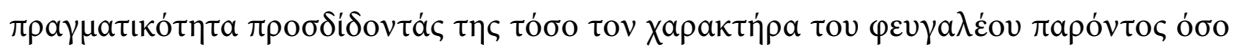

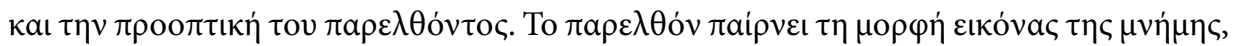

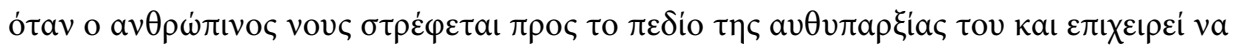

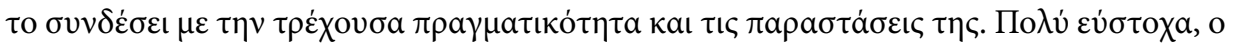

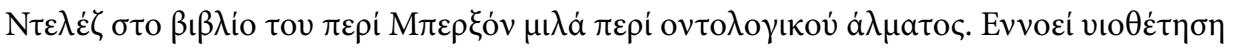

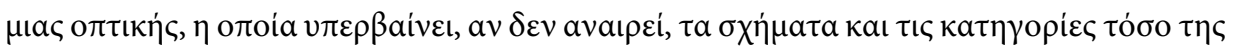

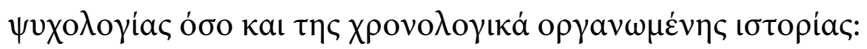

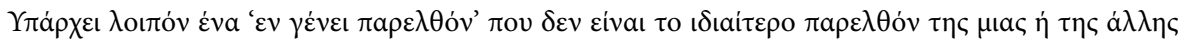

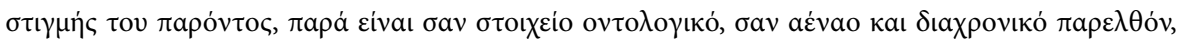

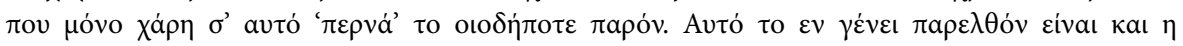

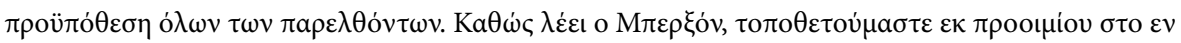

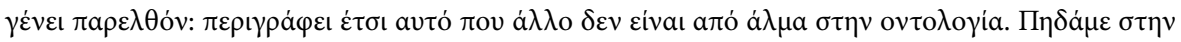

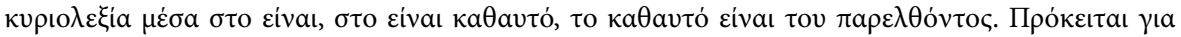

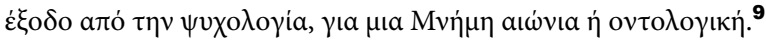

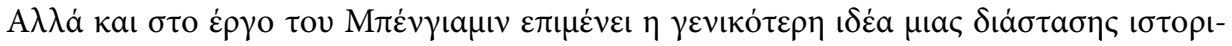

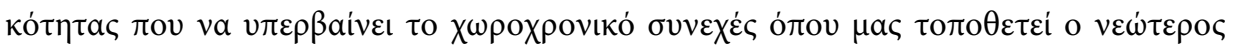

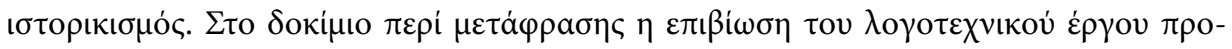

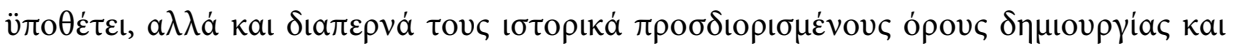

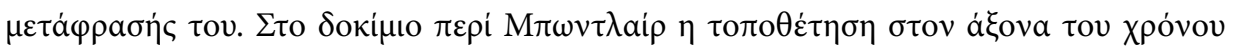

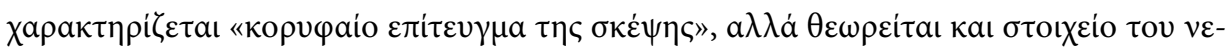

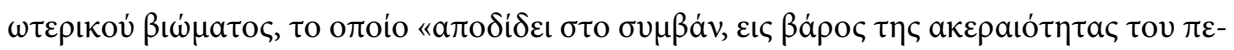

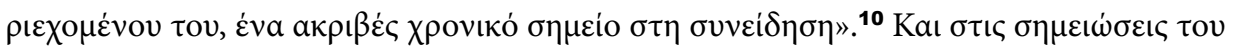

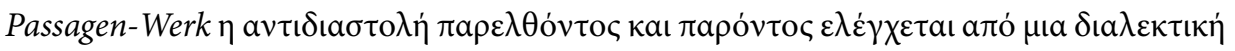

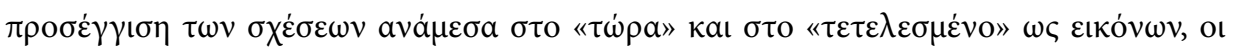

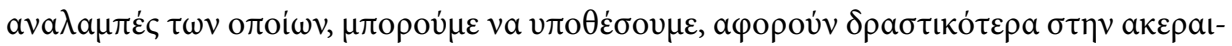

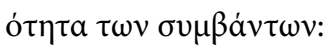

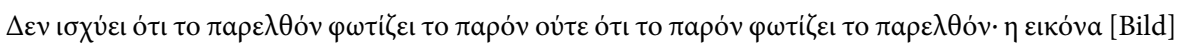

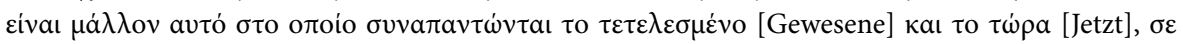

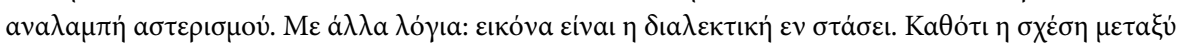

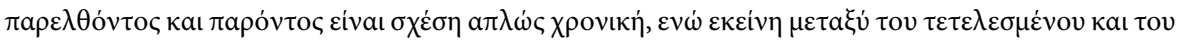

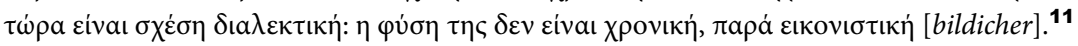

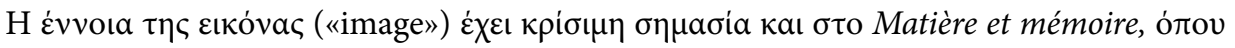

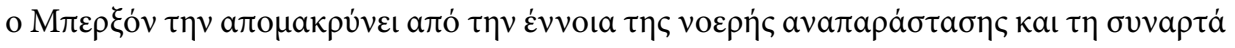

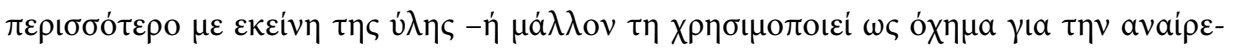

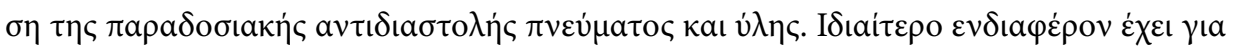




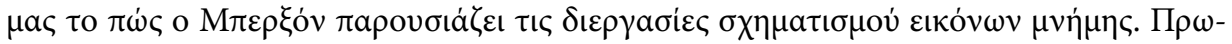

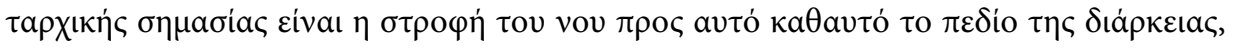

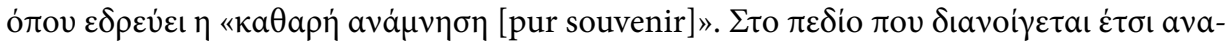

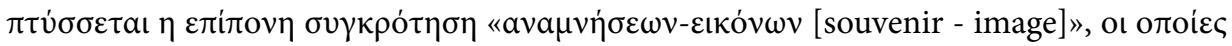

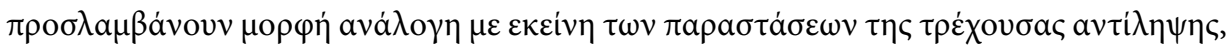

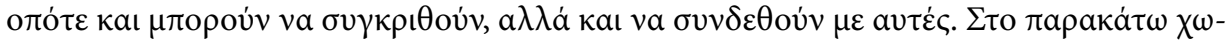

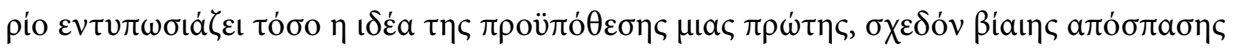

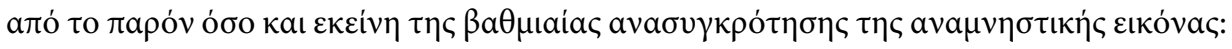

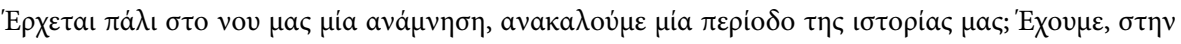

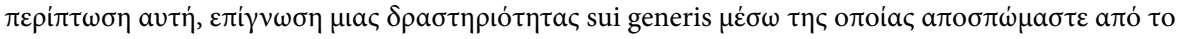

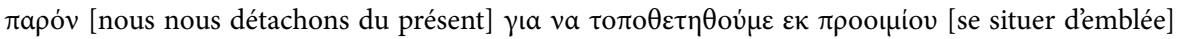

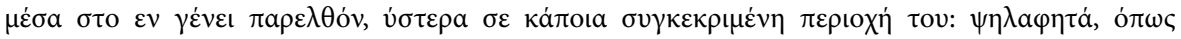

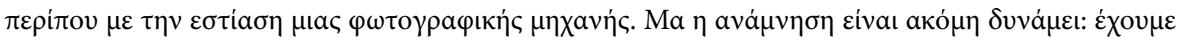

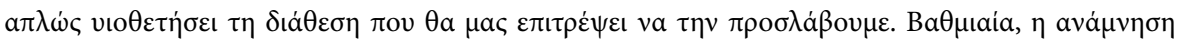

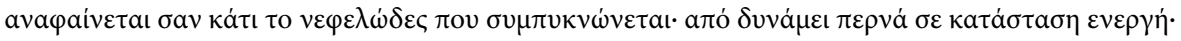

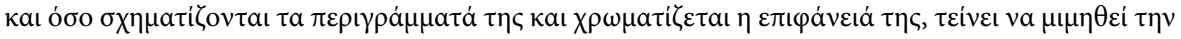

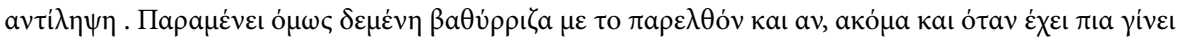

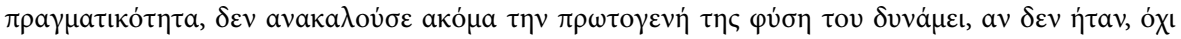

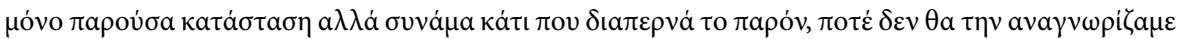
$\omega \varsigma$ a $\alpha \dot{\alpha} \mu \nu \eta \sigma \eta .^{12}$
\end{abstract}

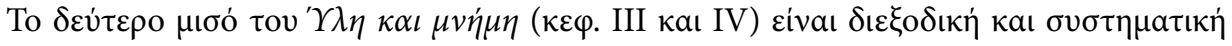

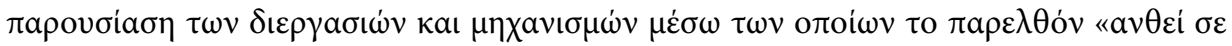

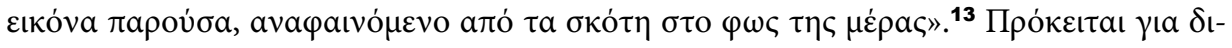

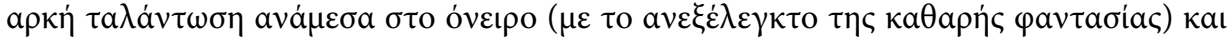

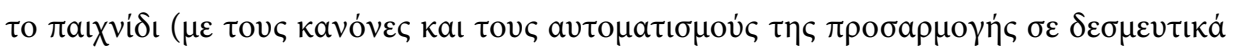

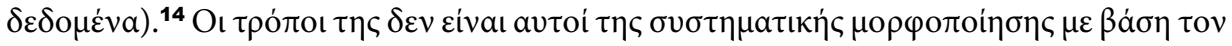

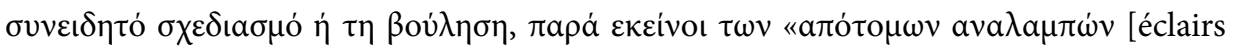

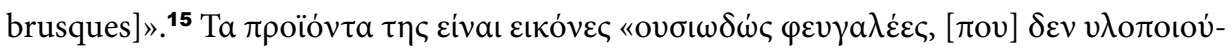

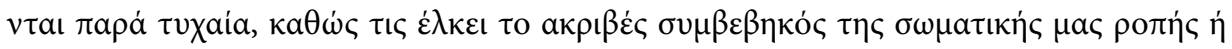

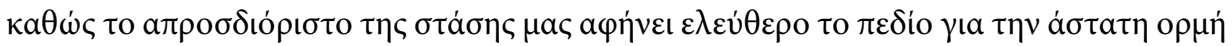

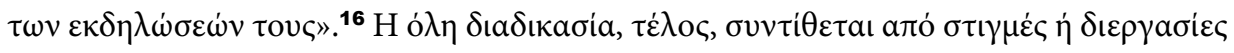

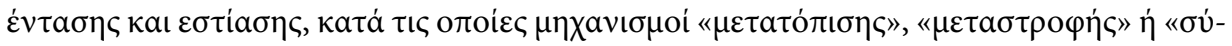

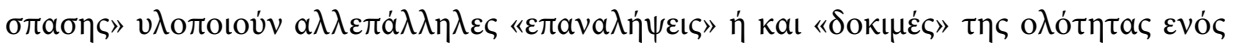

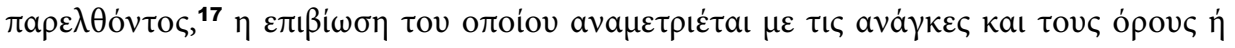

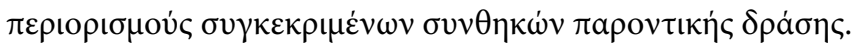

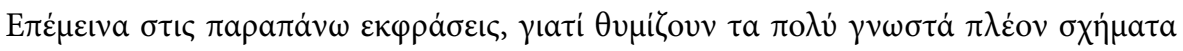

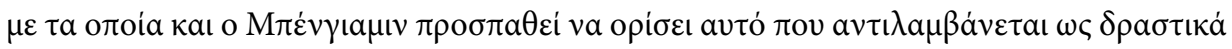

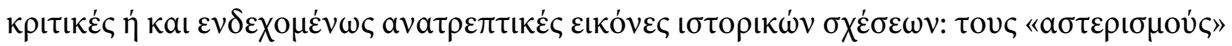




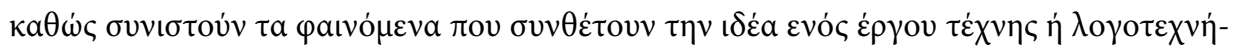

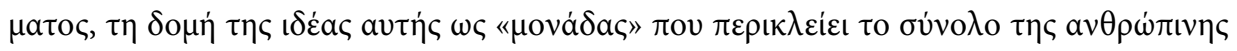

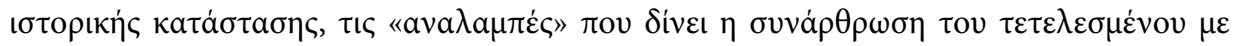

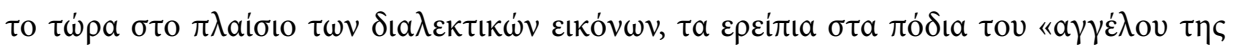

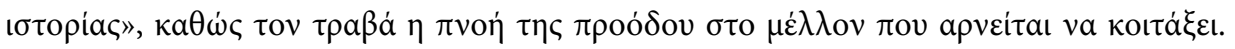

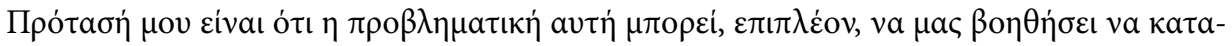

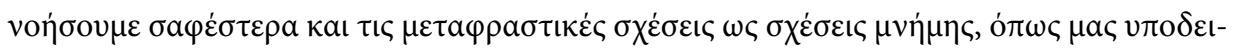

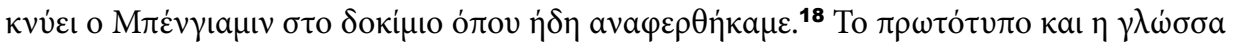

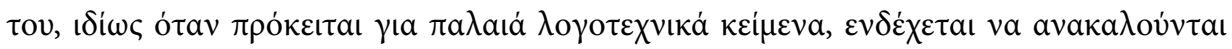

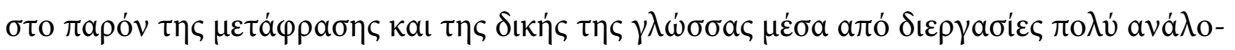

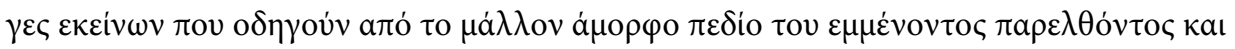

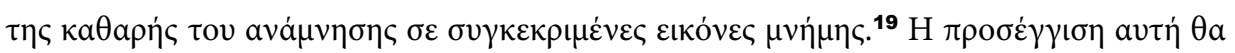

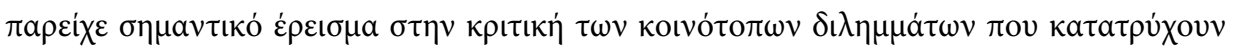

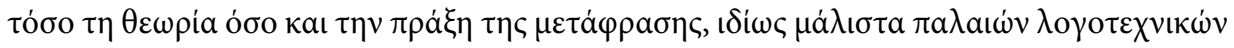

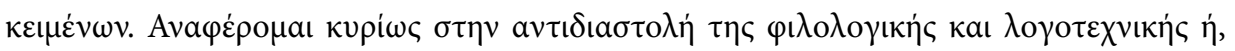

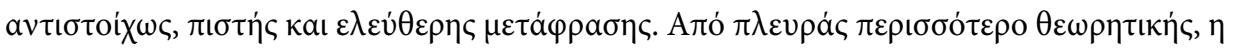

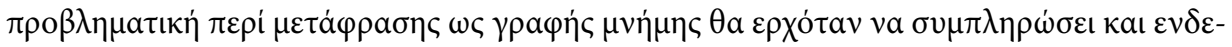

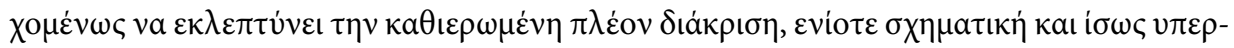

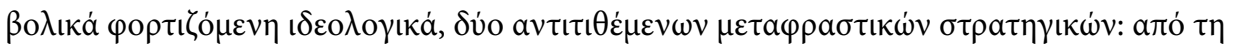

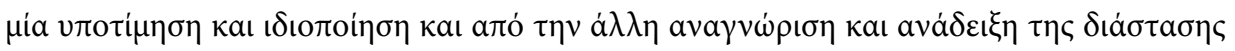

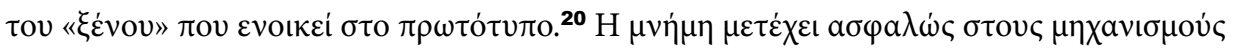

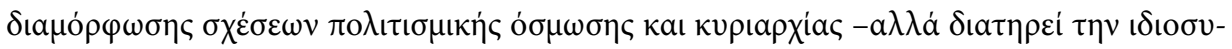

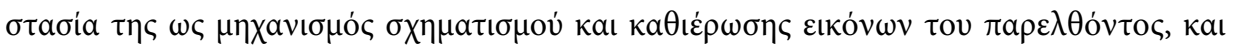

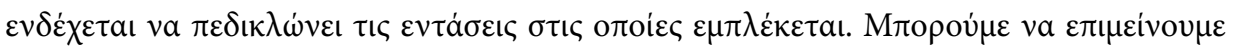

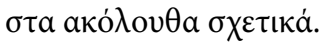

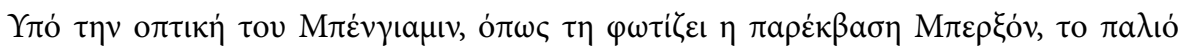

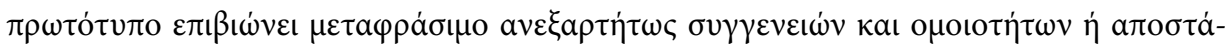

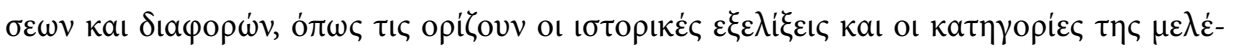

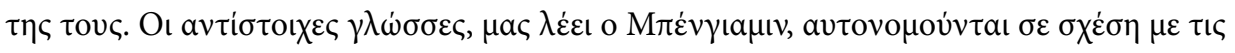

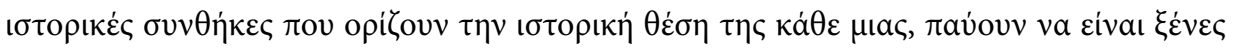

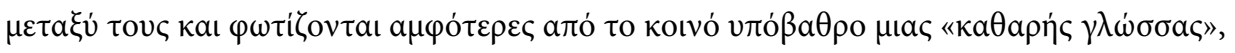

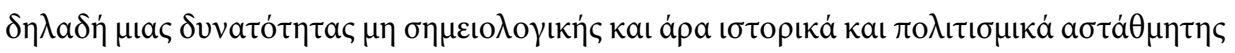

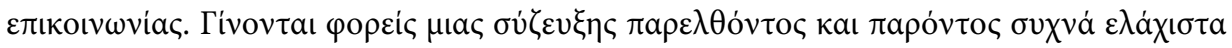

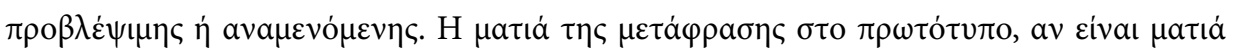

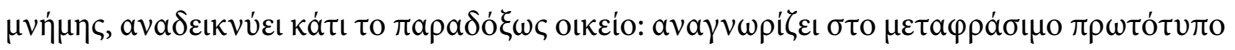

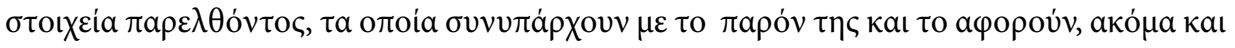

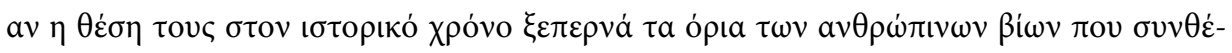




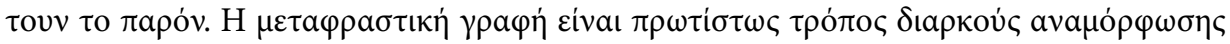

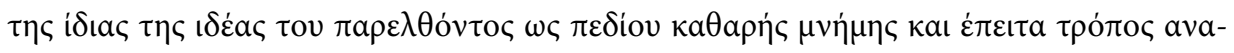

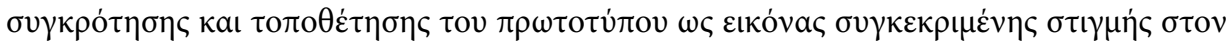

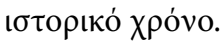

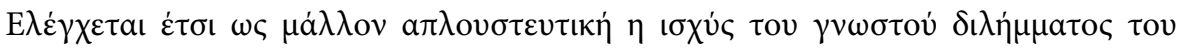

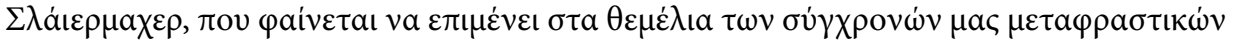

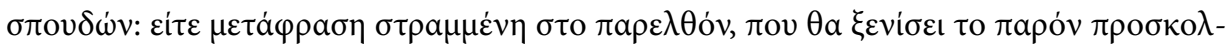

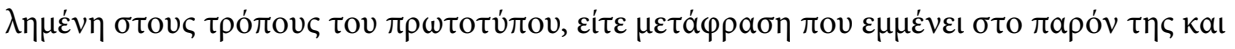

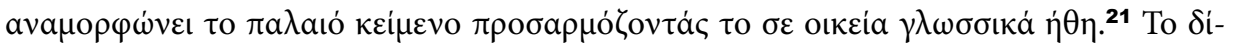

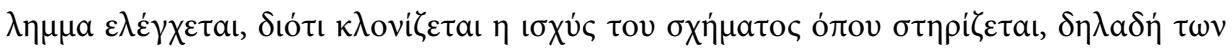

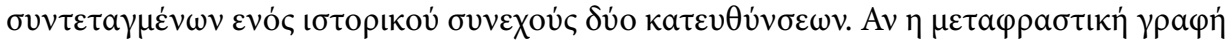

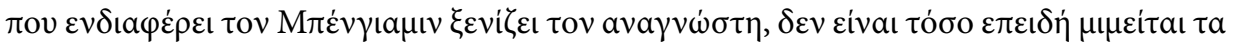

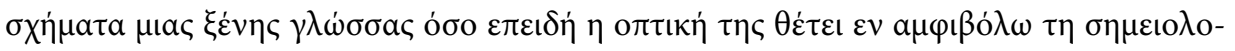

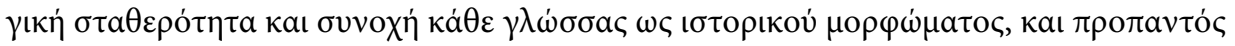

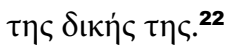

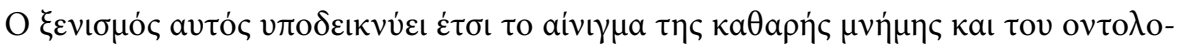

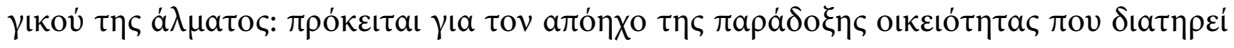

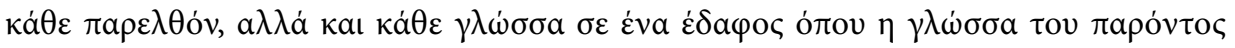

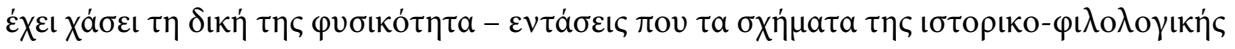
$\alpha v a ́ \lambda v \sigma \eta \varsigma$ a

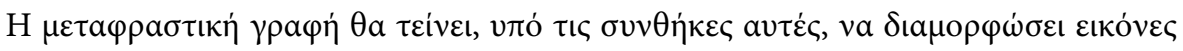

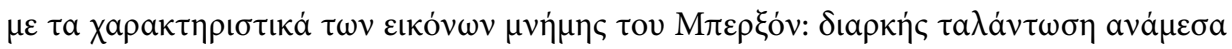

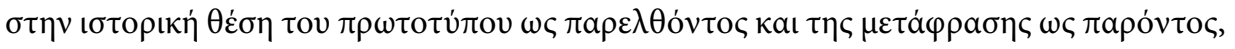

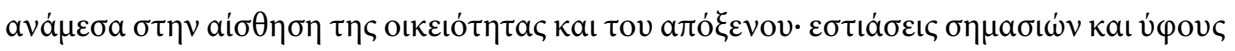

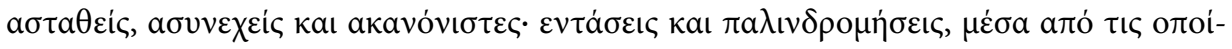

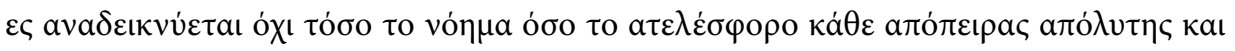

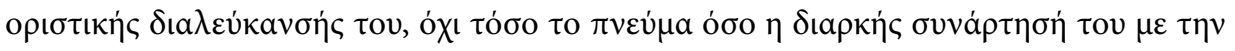

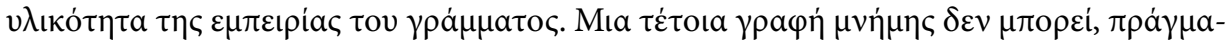

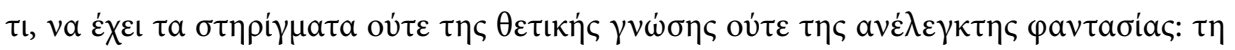

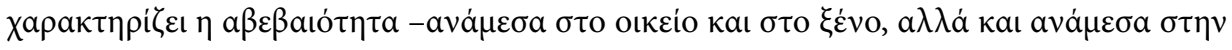

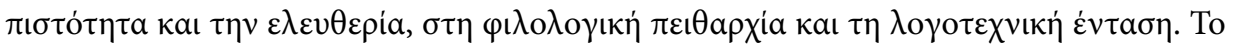

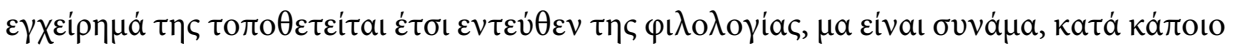

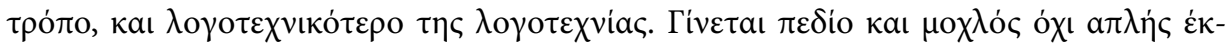

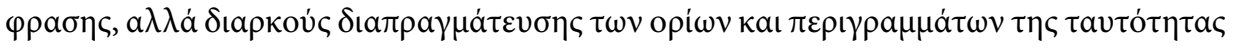

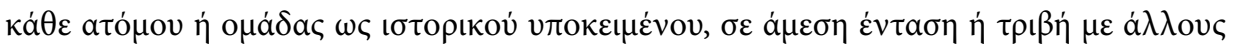

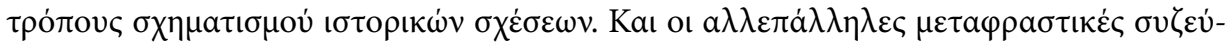

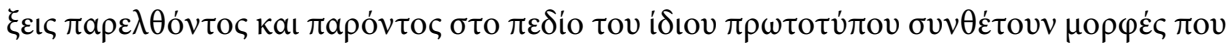




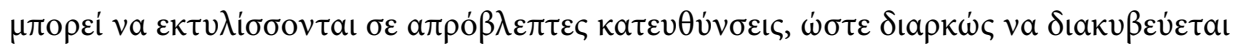

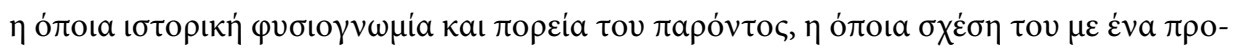

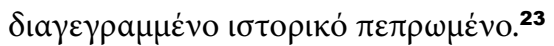

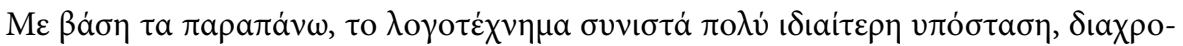

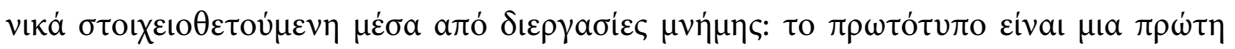

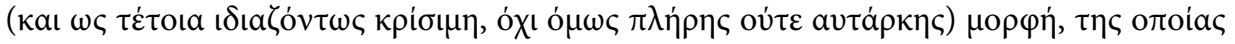

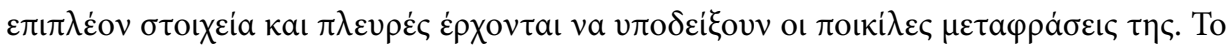

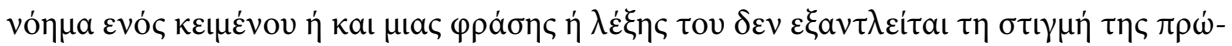

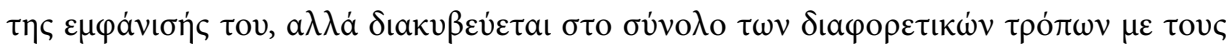

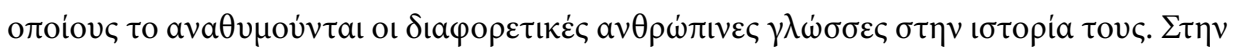

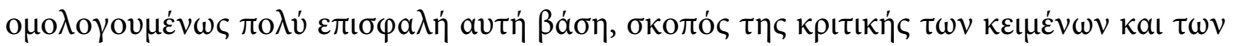

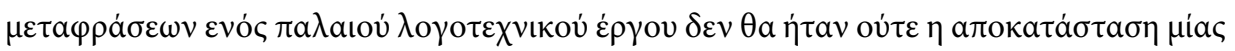

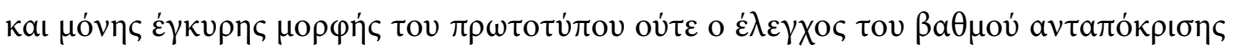

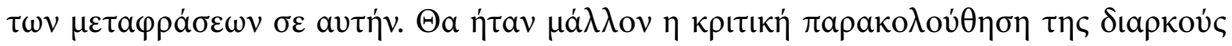

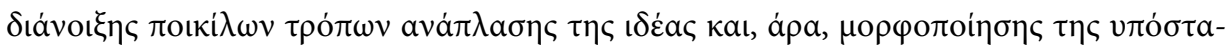

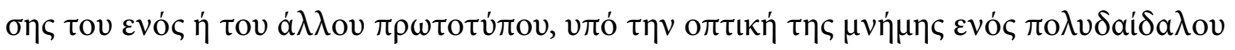

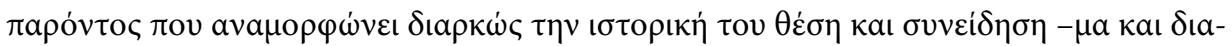

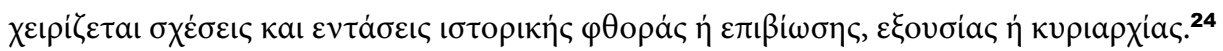

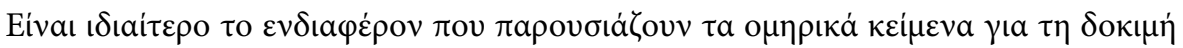

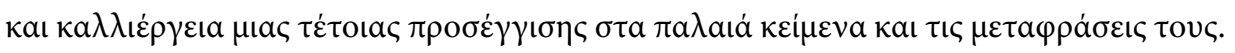

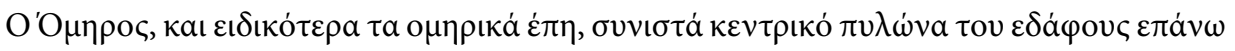

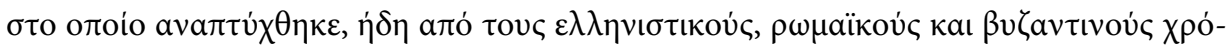

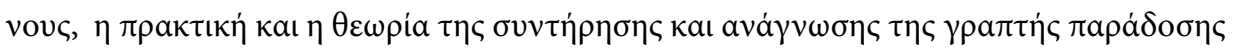

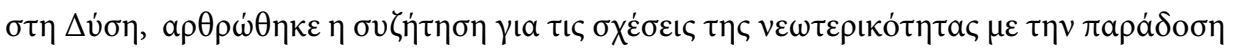

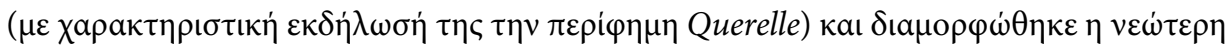

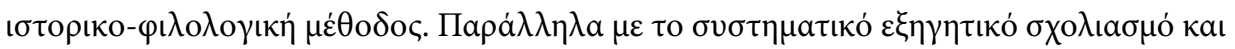

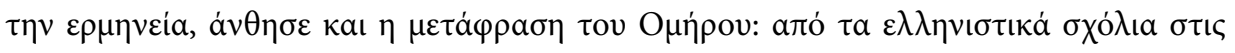

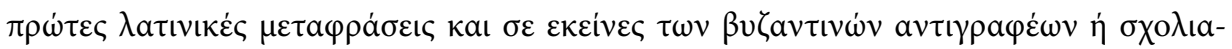

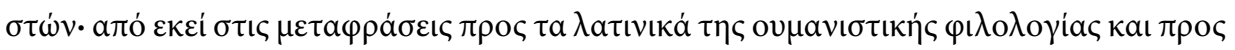

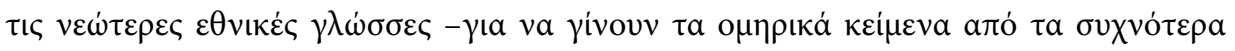

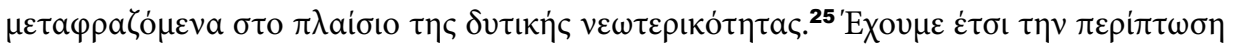

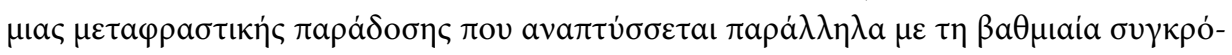

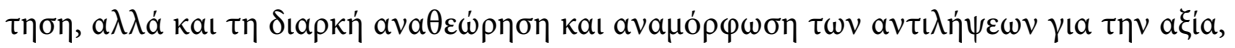

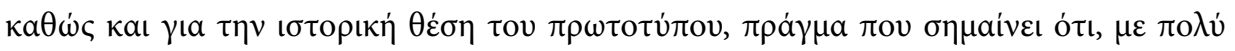

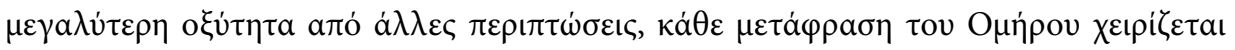

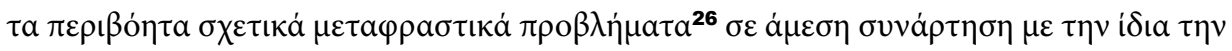

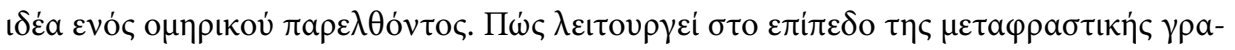




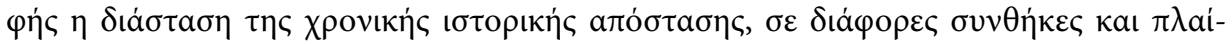

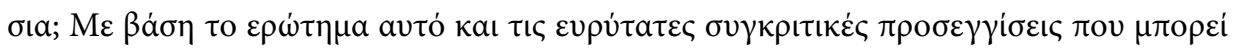

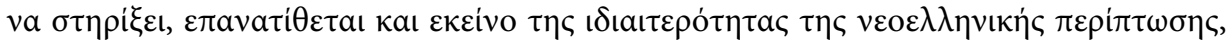

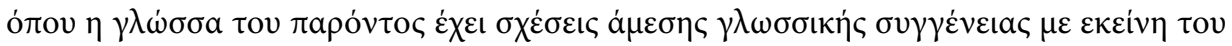

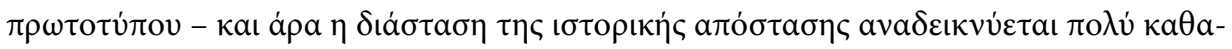

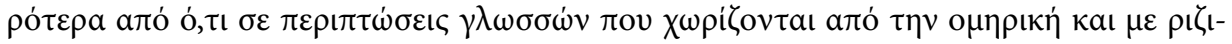

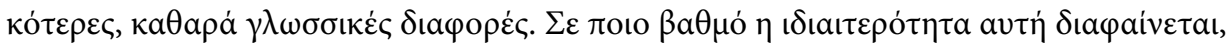

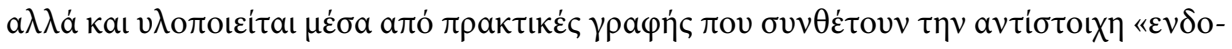

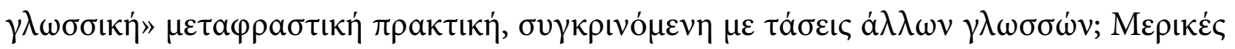

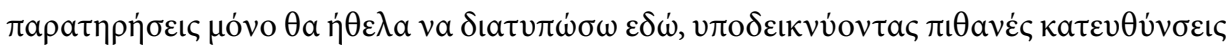

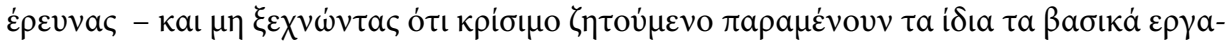

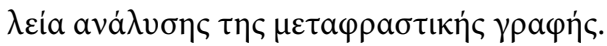

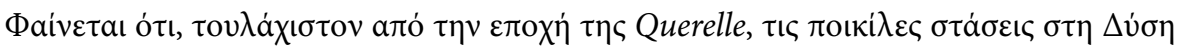

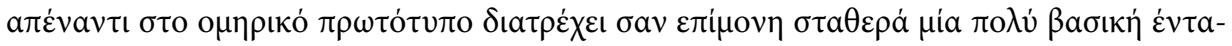

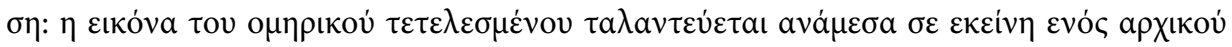

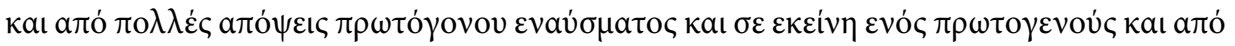
$\pi 0 \lambda \lambda \dot{\varepsilon} \varsigma$ a

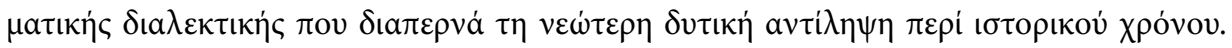

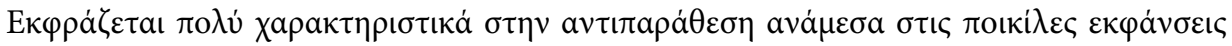

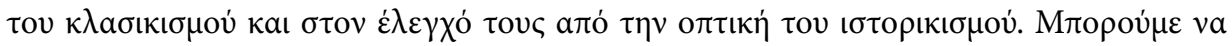

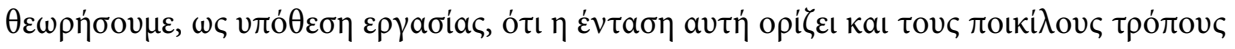

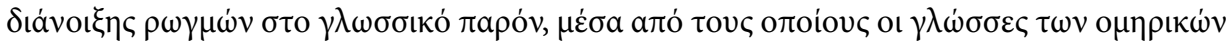

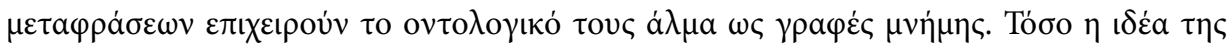

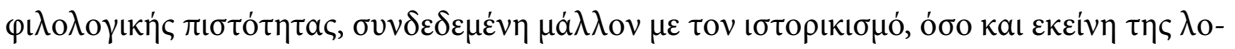

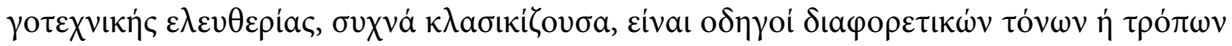

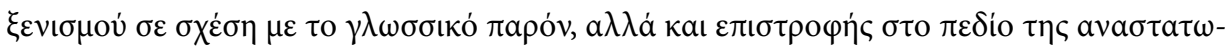

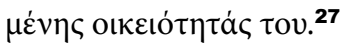

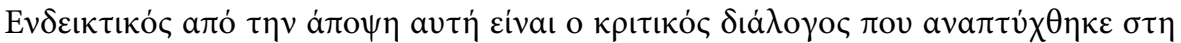

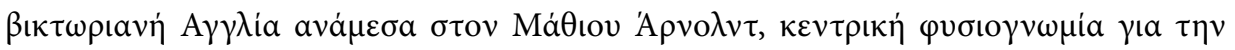

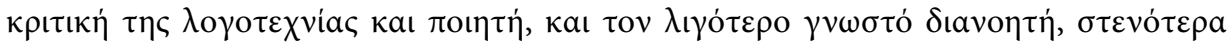

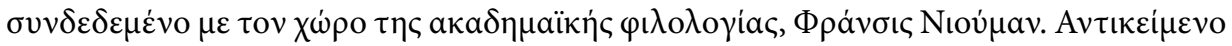

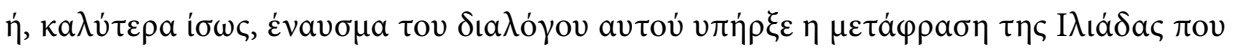

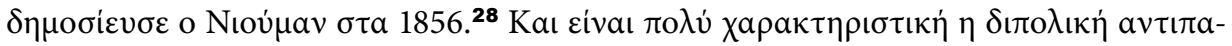

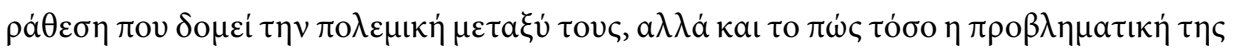

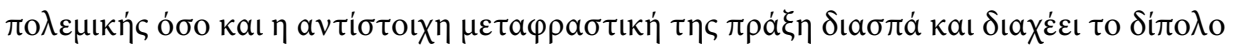

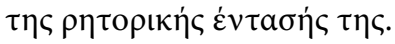




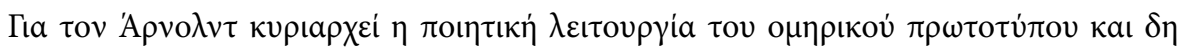

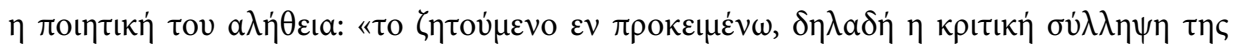

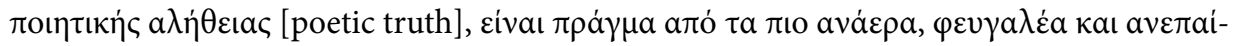

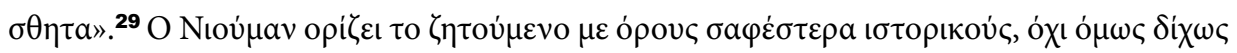

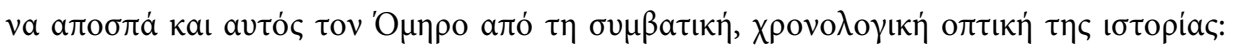

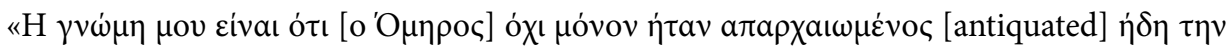

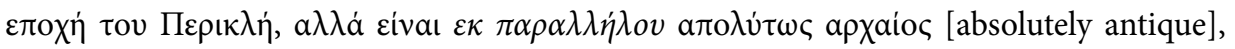

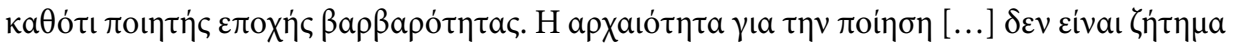

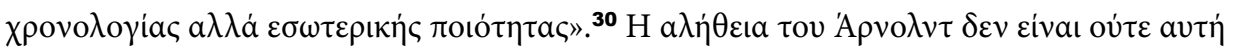

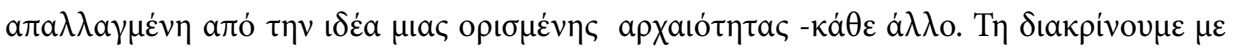

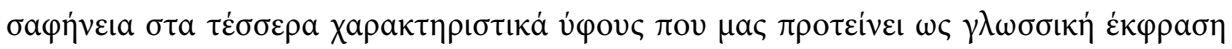

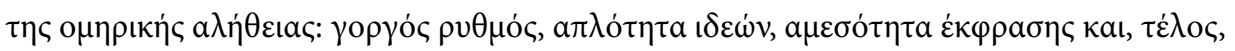

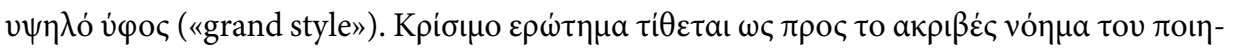

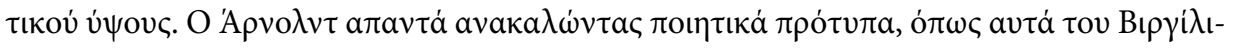

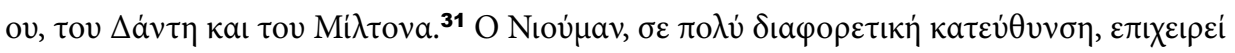

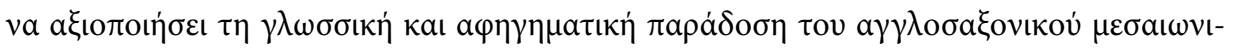

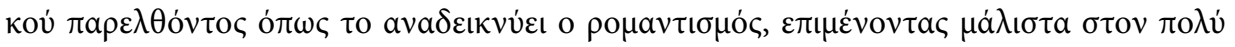

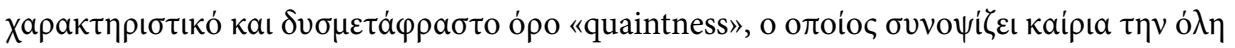

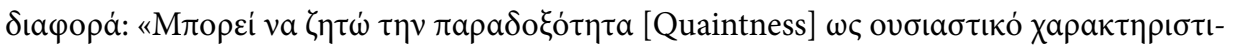

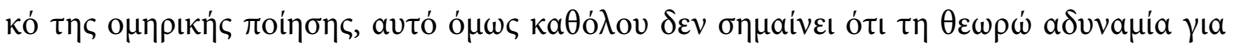

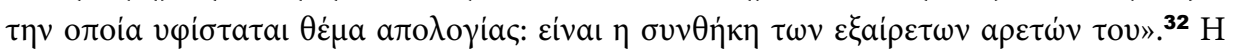

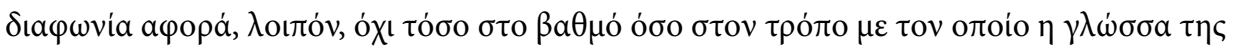

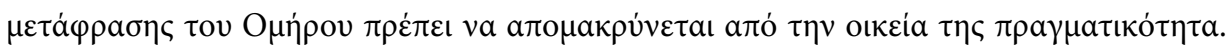

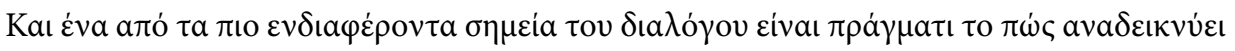

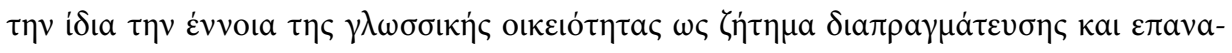

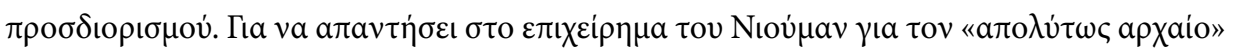

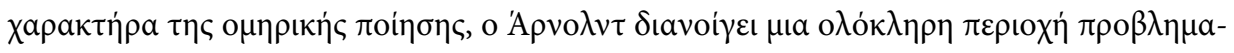

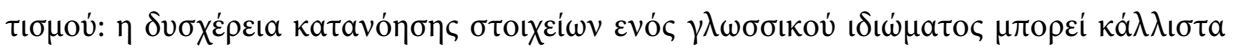

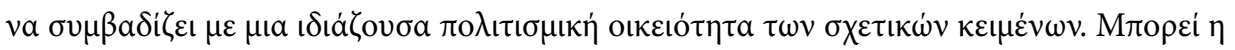

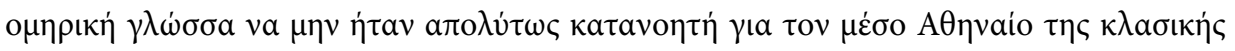

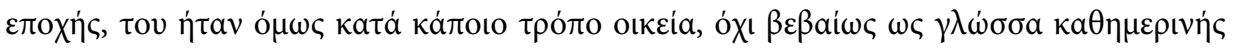

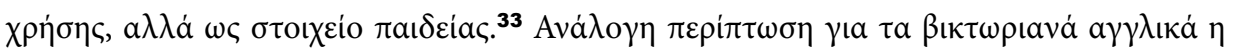

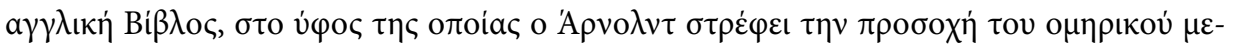

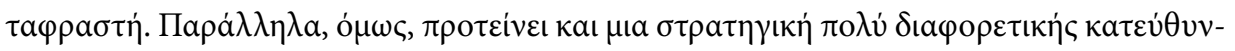

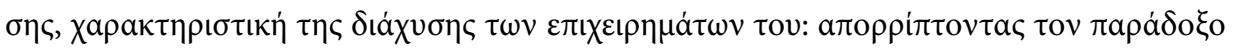

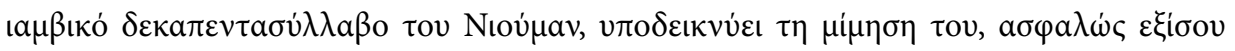

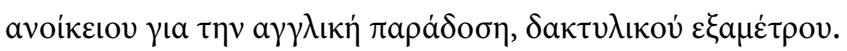




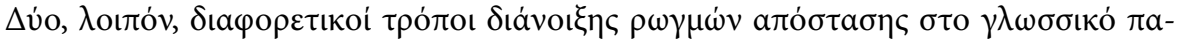

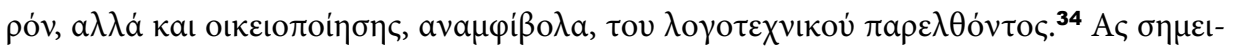

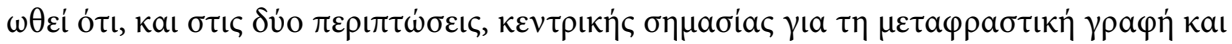

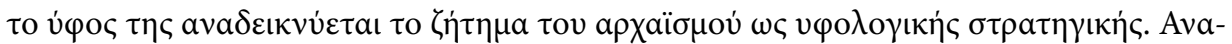

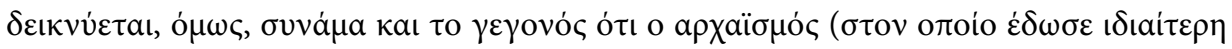

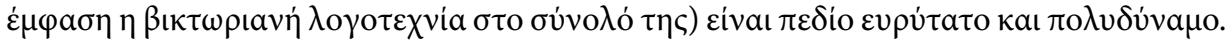

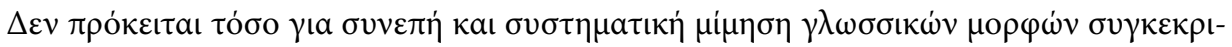

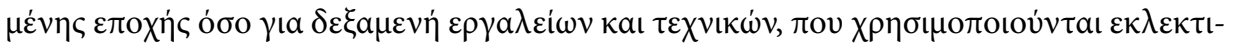

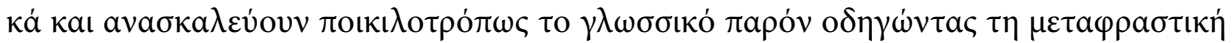

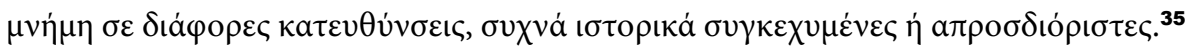

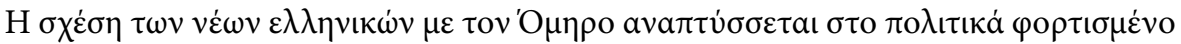

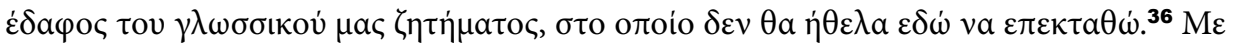

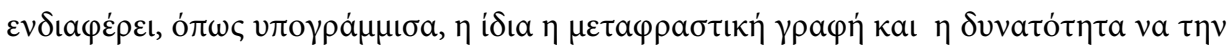

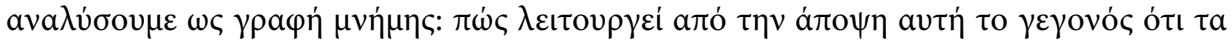

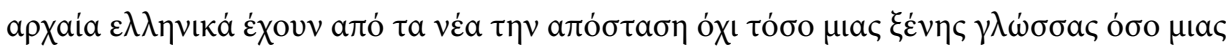

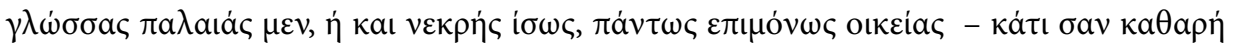

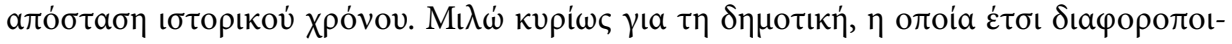

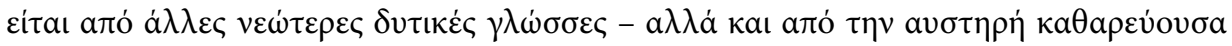

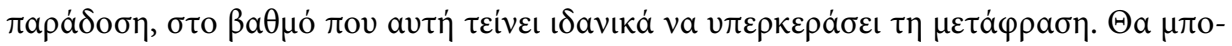

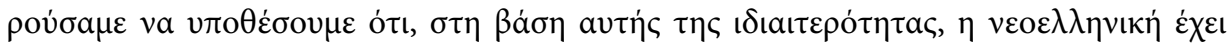

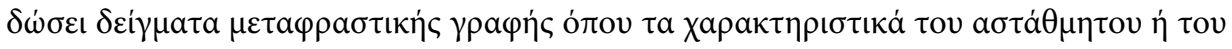

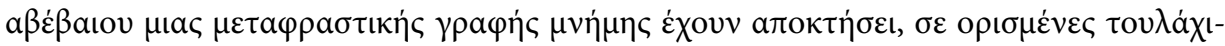

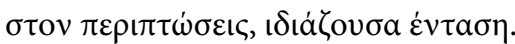

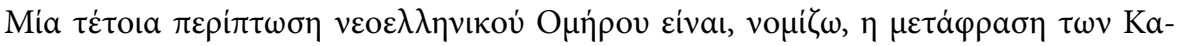

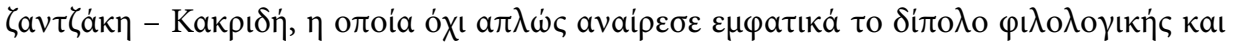

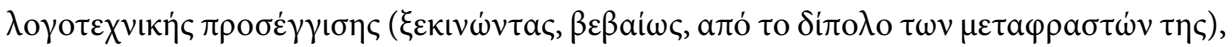

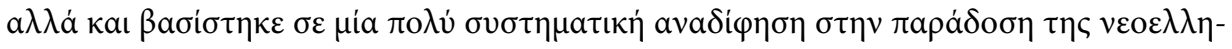

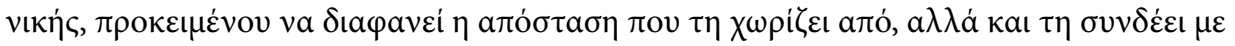

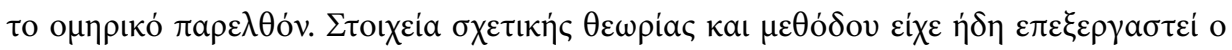

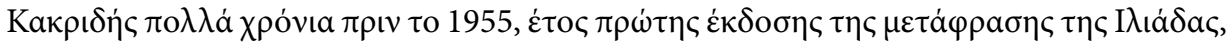

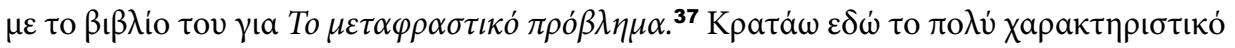

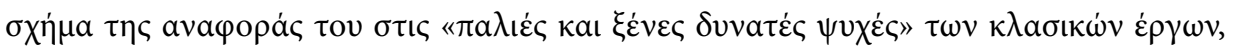

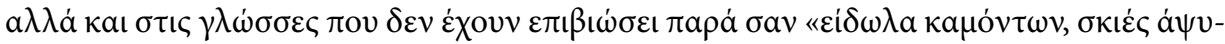

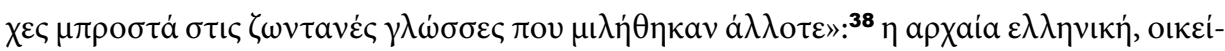

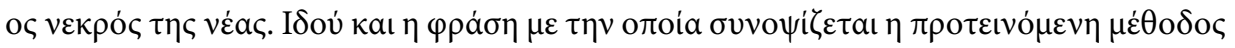

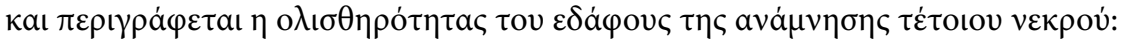




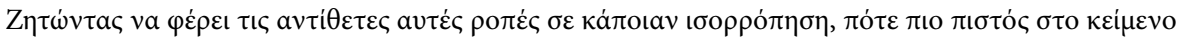

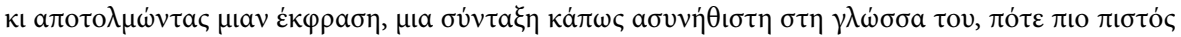

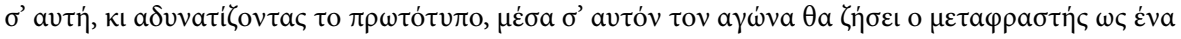

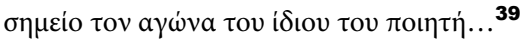

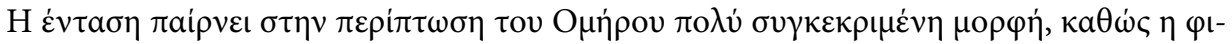

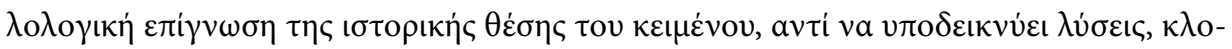

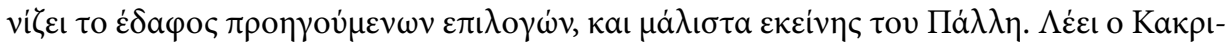

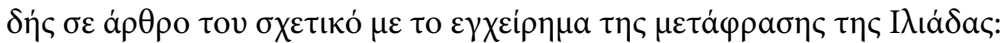

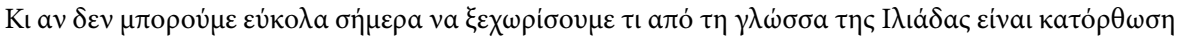

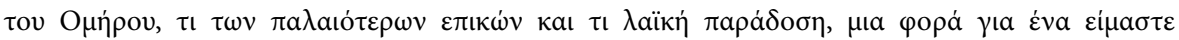
б́́ $\tau \rho a \gamma o v \delta ı \tau \omega \dot{\omega} v^{\mathbf{4 0}}$

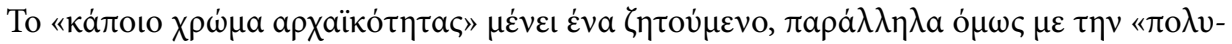

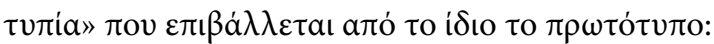

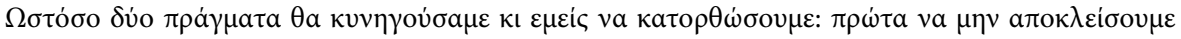

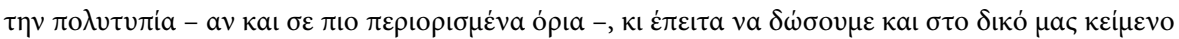

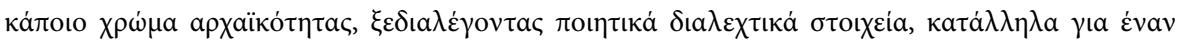

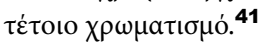

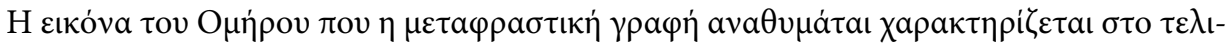

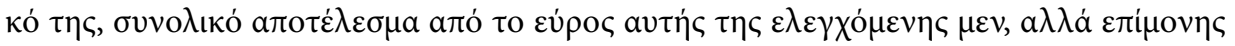

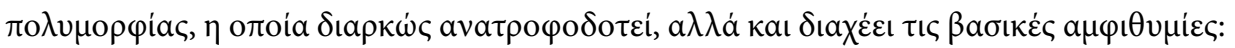

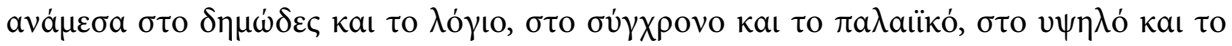

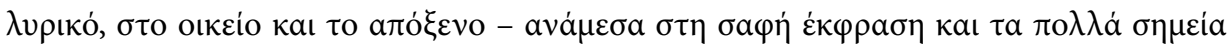

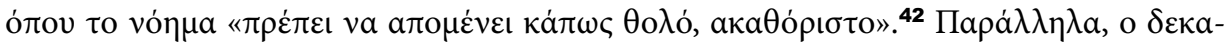

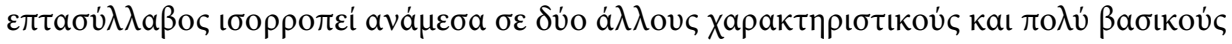

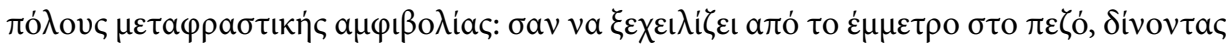

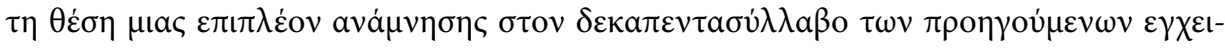

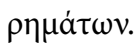

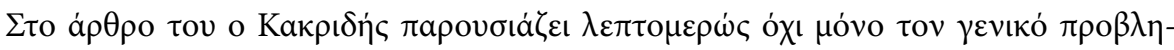

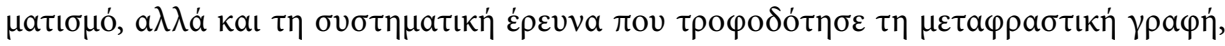

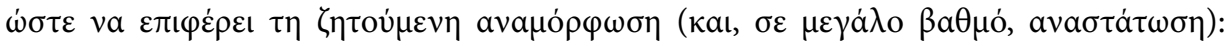

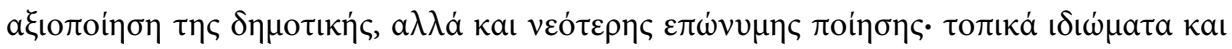

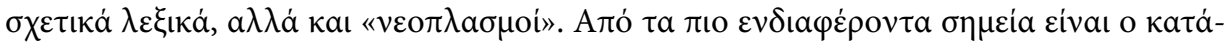

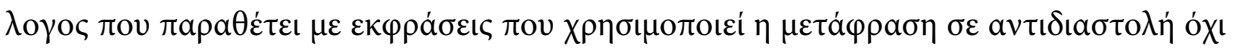

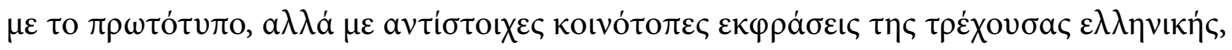

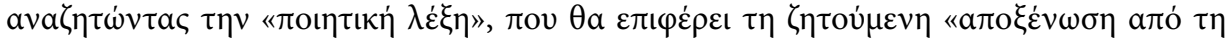

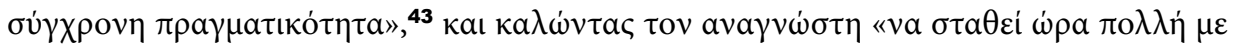




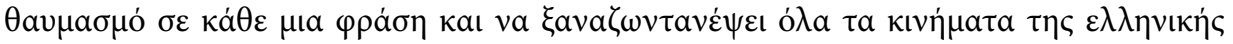

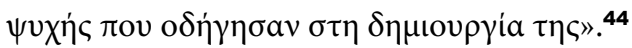

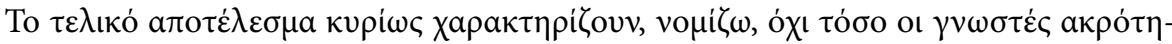

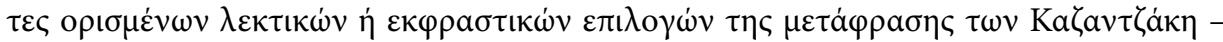

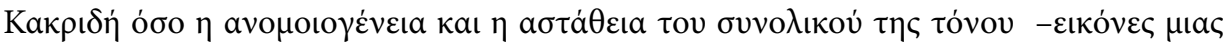

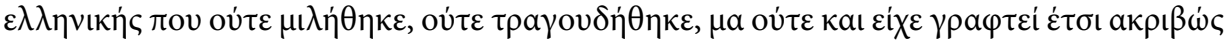

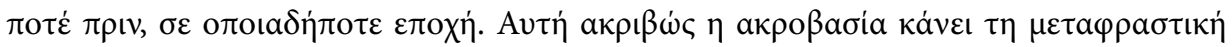

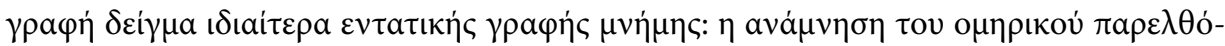

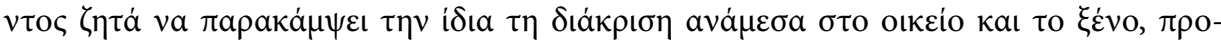

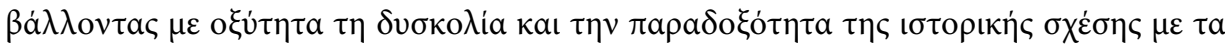

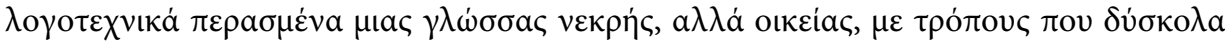

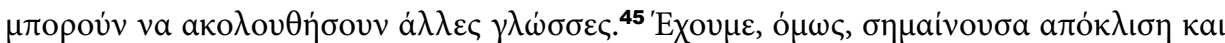

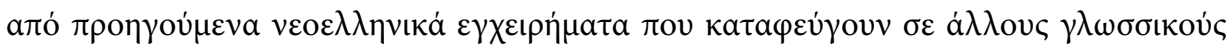

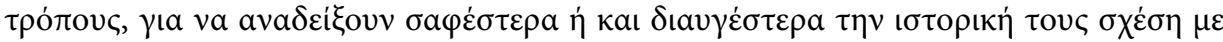

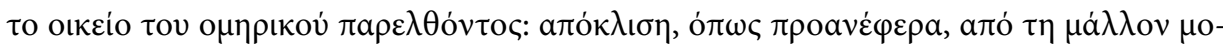

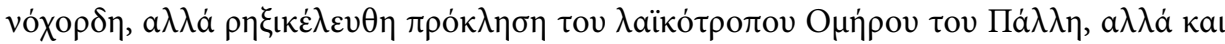
a

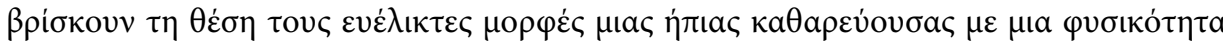

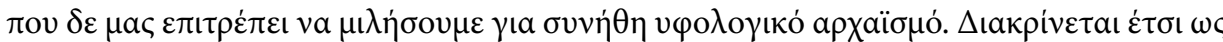

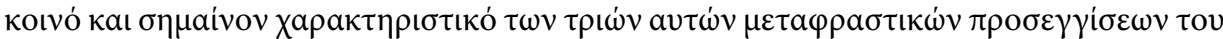

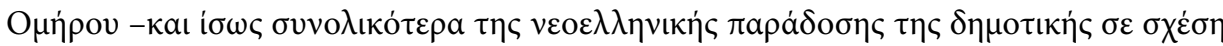

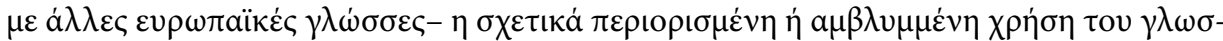

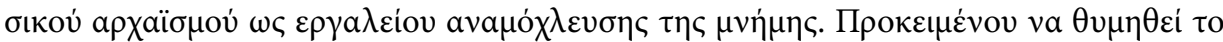

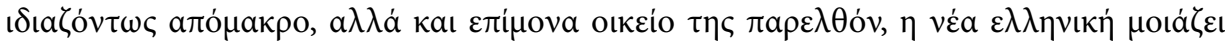

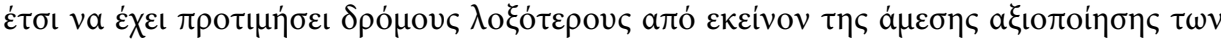

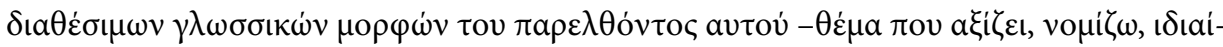
$\tau \varepsilon \rho \eta \pi \rho \circ \sigma o \chi \eta \dot{~}{ }^{46}$

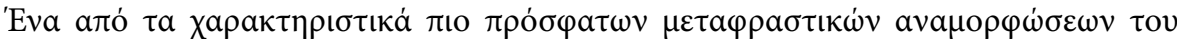

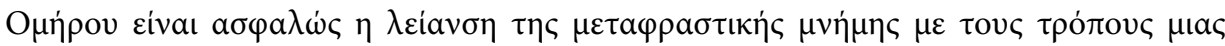

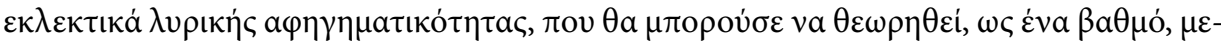

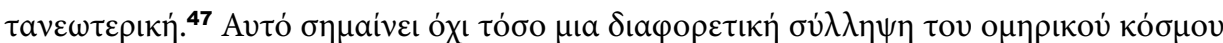

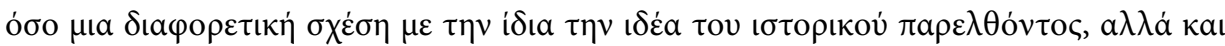

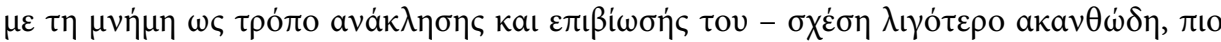

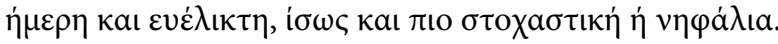




\section{ПАРАРТНМА}

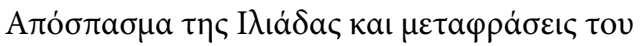

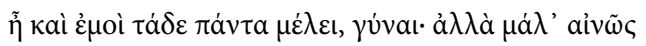

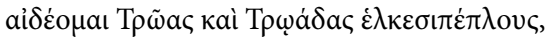

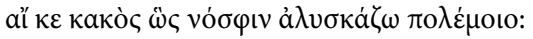

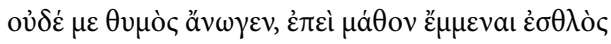

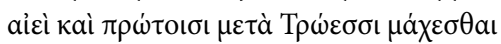

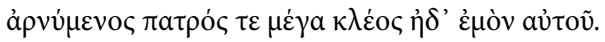

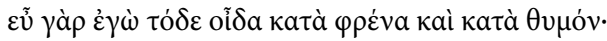

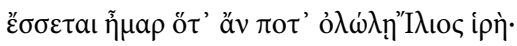

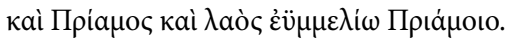

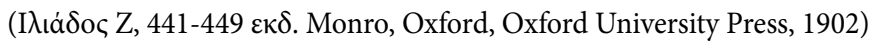

O lady, all this argument by me is too regarded:

But direfully abash'd were I before the men of Troas And Troian women trailing-rob'd, if, skulking like a coward, I shunn'd the war: not doth my heart allow it; but hath taught me Mid foremost Troians to fight and show a chieftain's virtue, And guard my father's great renown and lift myself to glory. for this in heart and soul full sure I know; a day is coming A day, when sacred Ilium for overthrow is destin'd, and Priam's self, and the folk of ashen-speared Priam.

(Francis Newman: The Iliad of Homer, London, Walton and Maberly, 1856)

Woman, I too take thought for this; but then I bethink me What the Trojan men and Trojan women might murmur, If like a coward I skulked behind, apart from the battle. Nor would my own heart let me; my heart, which has bid me valiant Always, and always fighting among the first of Trojans, busy for Priam's fame and my own, in spite of the future. For that day will come, my soul is assured of its coming, it will come, when sacred Ilium shall go to destruction, Troy and warlike Priam too, and the people of Priam.

(Matthew Arnold: aró to «Translating Homer», in On the Classical Tradition, Ann Arbor, The University of Michigan Press, 1960, б. 164) 


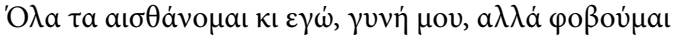

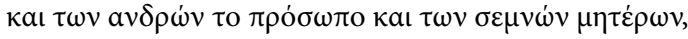

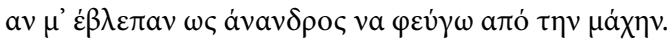

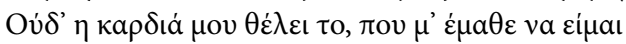

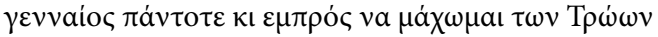

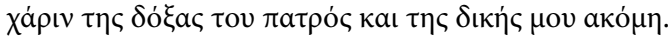

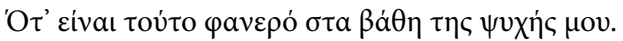

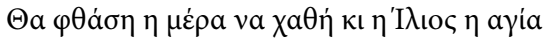

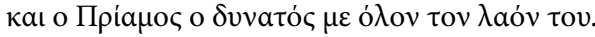

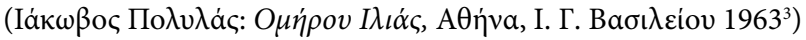

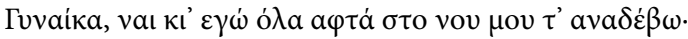

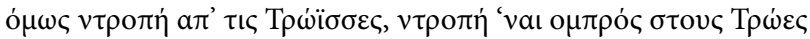

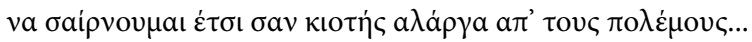

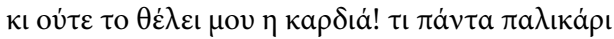

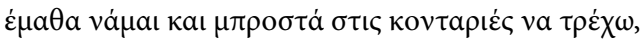

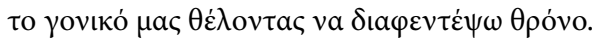

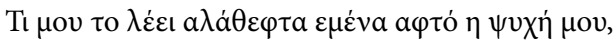

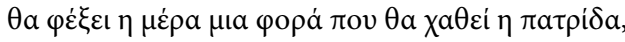

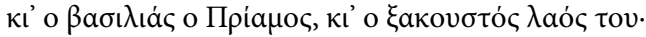

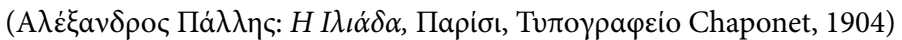

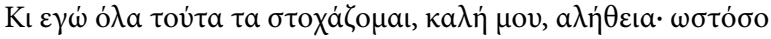

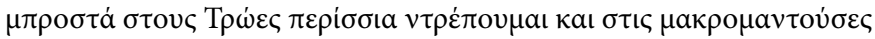

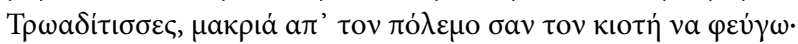

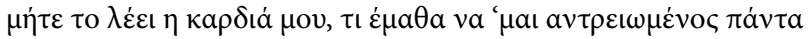

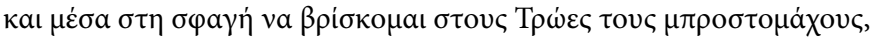

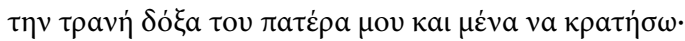

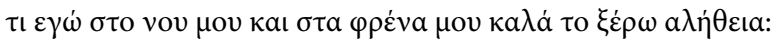

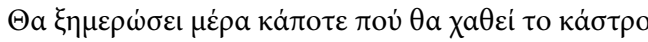

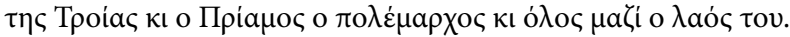

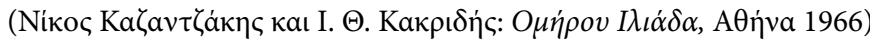




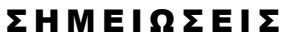

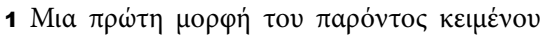

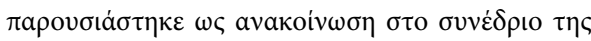

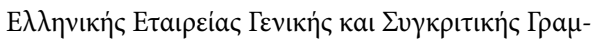

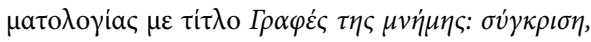

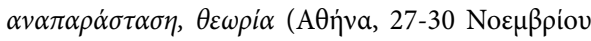

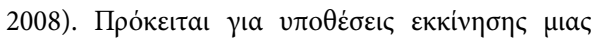

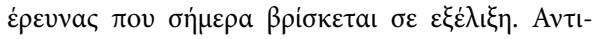

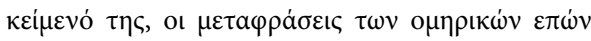

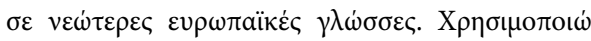

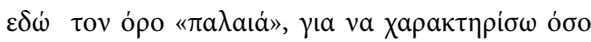

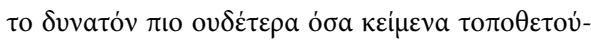

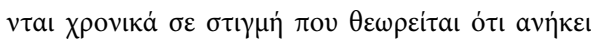

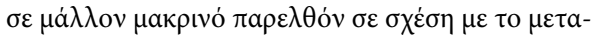

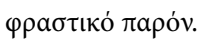

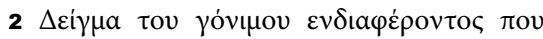

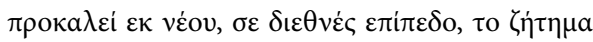

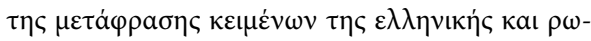

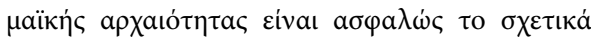

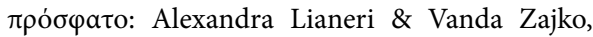
Translation and the Classic: Identity as Change in the History of Culture, Oxford, Oxford University Press, 2008.

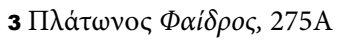

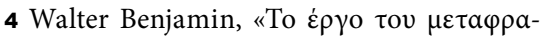

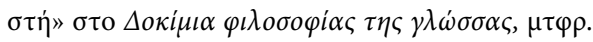

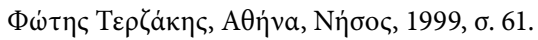

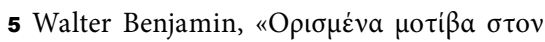

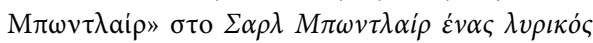

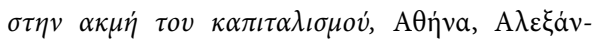

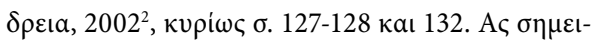

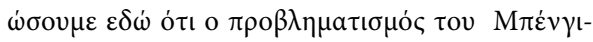

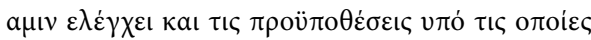

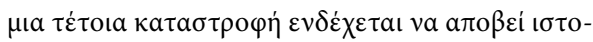

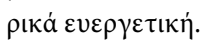

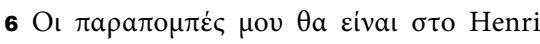
Bergson, Matière et mémoire: Essai sur la relation $d u$ corps à l'esprit, PUF : Paris, Quadrige Presses Univesitaires de France, 1939 [a

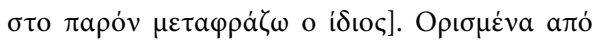

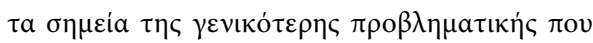

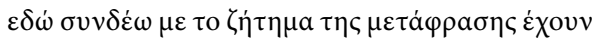

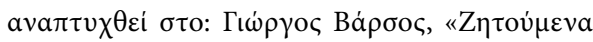

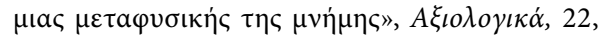

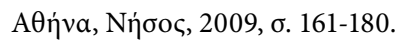

7 Walter Benjamin, ó. $\pi ., \sigma .125$.

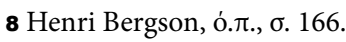

9 Gilles Deleuze, Le Bergsonisme, Presses Univesitaires de France, Paris, 1966, б. 52.

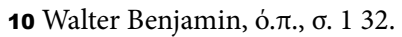

11 Walter Benjamin, Gesammelte Schriften, V, I, Frankfurt am Main, Suhrkamp Verlag, 1982,

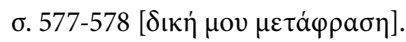

12 Henri Bergson, ó. $\pi$., $\sigma .148$.

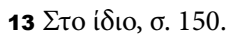

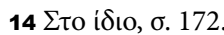

$15 \Sigma \tau$ 七 í̊ı, б. 93.

$16 \Sigma$ to í $\delta$ เo, $\sigma .116$.

$17 \sum \tau$ to ísı, o. 188.

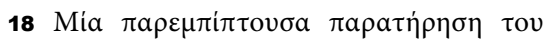

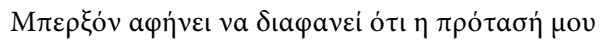

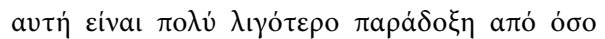

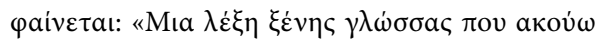

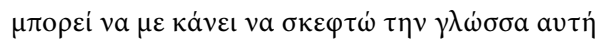

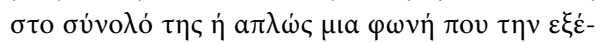

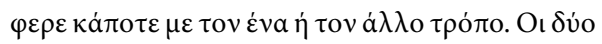

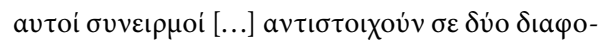

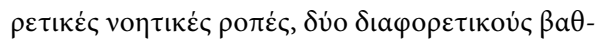

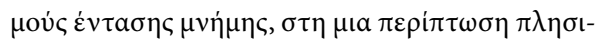
$\dot{\varepsilon} \sigma \tau \varepsilon \rho \eta \sigma \tau \eta v \kappa \alpha \theta \alpha \rho \eta \dot{~ \varepsilon เ \kappa o ́ v a, ~ \sigma \tau \eta v ~ a ́ \lambda \lambda \eta \eta ~ \pi \varepsilon \rho ı \sigma \sigma o ́-~}$ $\tau \varepsilon \rho \circ \sigma v v \delta \varepsilon \delta \varepsilon \mu \varepsilon \dot{v} \eta \eta \mu \varepsilon \dot{\alpha} \mu \varepsilon \sigma \eta \alpha v \tau i \delta \rho a \sigma \eta, \delta \eta \lambda \alpha \delta \dot{\eta}$

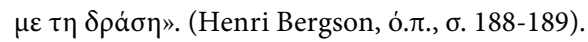

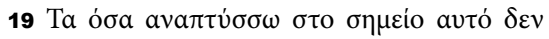

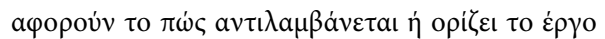

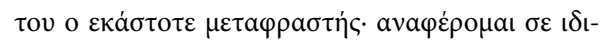

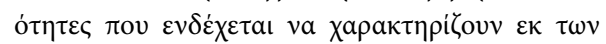

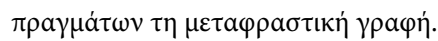

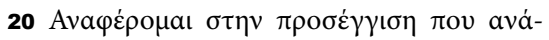

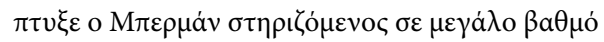

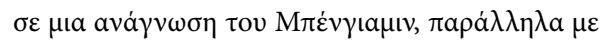

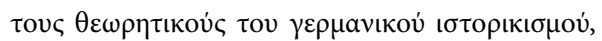

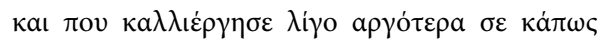

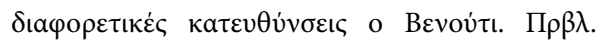
Antoine Berman, La Traduction et la lettre ou l'Auberge du lointain, Paris, Editions du Seuil, 1999 кa।

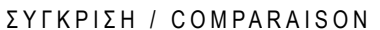

$22(2011)$ 
Lawrence Venuti, The Translator's Invisibility: A History of Translation, London, Routledge, 1995.

$21 \mathrm{~B} \lambda$. Friedrich Schleiermacher, Des différentes méthodes de traduire, Paris, Éditions du

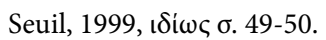

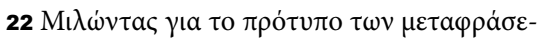

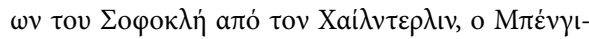

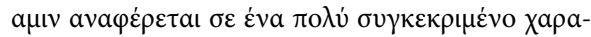

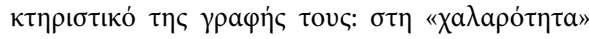

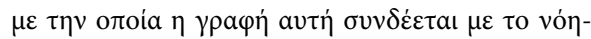

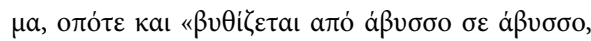

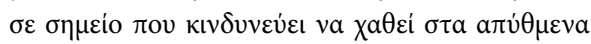

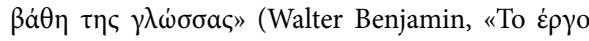

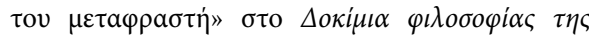

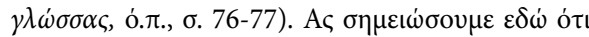

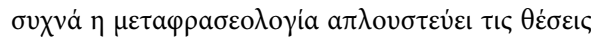

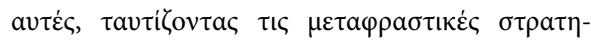

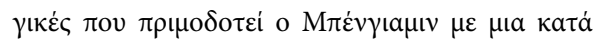

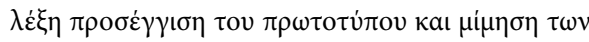

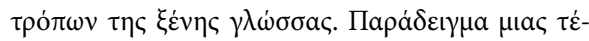

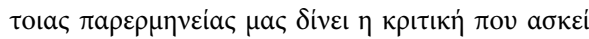

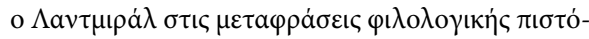

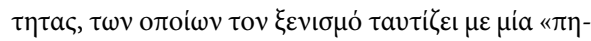

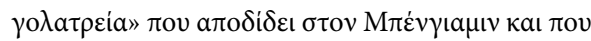

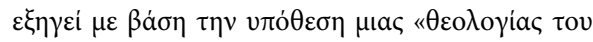

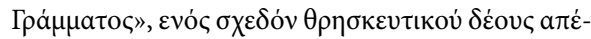

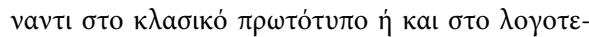

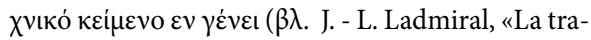
duction: des textes classiques?» $\sigma \tau$ o $S$. Nicosia (dir.) La traduzione dei testi classici. Teoria, prassi, storia, Atti del Convegno di Palermo, 6-9 Aprile 1988,

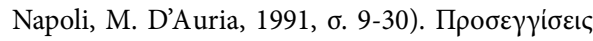

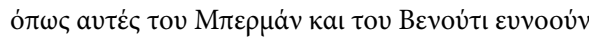

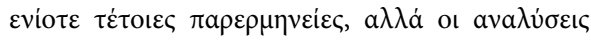

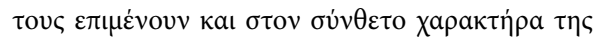

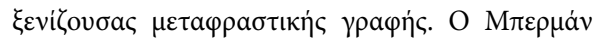

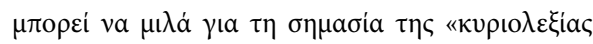
[littéralité]» $\sigma \tau \eta \quad \delta \eta \mu о v \rho \gamma \iota \kappa \eta ่ ~ \mu \varepsilon \tau \alpha \dot{\varphi} \varphi \rho \alpha \sigma \eta, a \lambda \lambda \dot{a}$

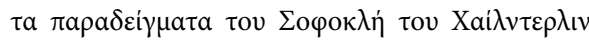

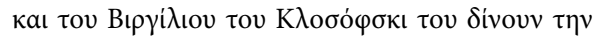

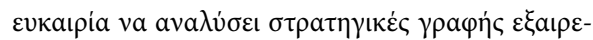

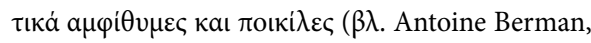

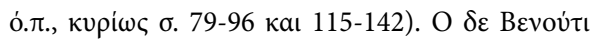

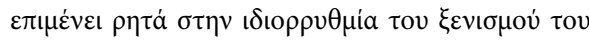

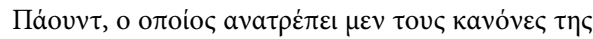

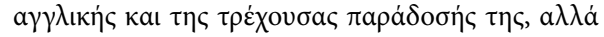

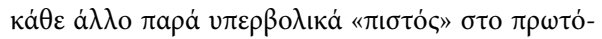

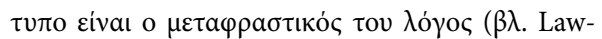

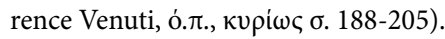

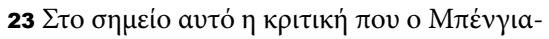

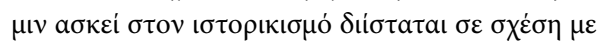

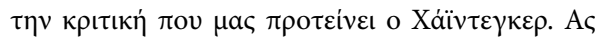

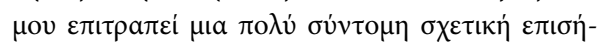

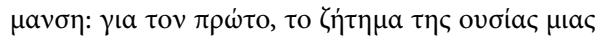

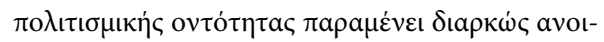

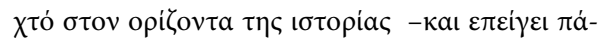

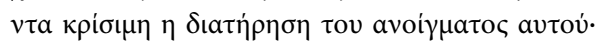

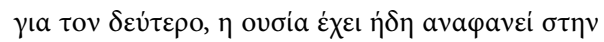

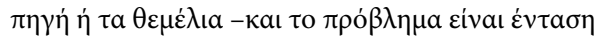

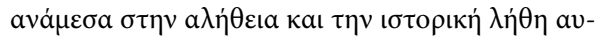

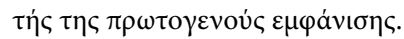

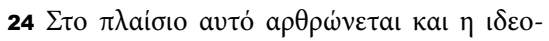

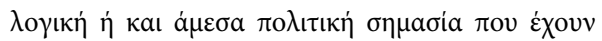

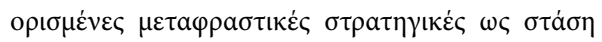

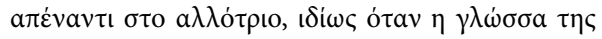

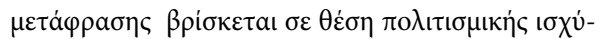

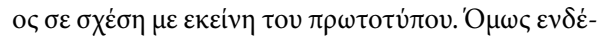

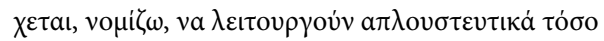

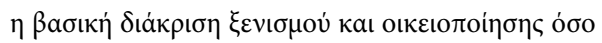

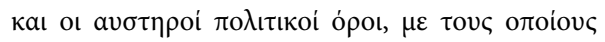

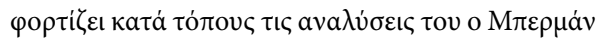

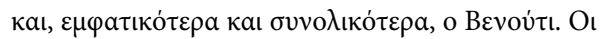
ó

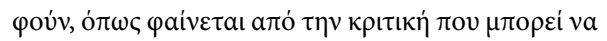

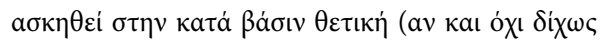

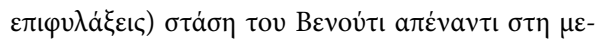

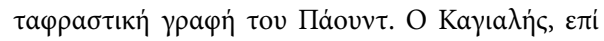

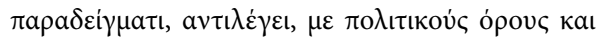

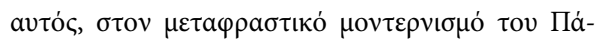

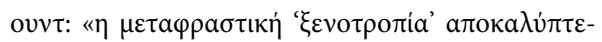

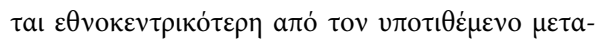

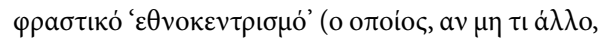

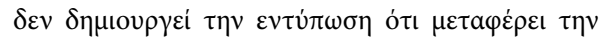

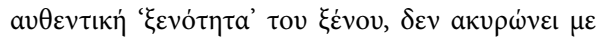

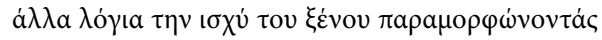

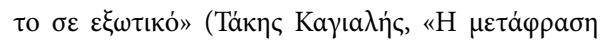

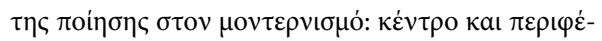

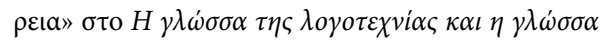

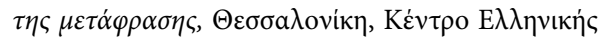

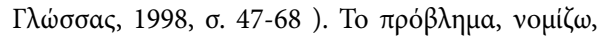




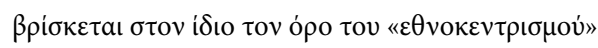

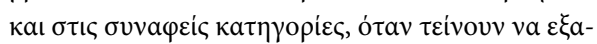

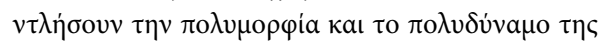

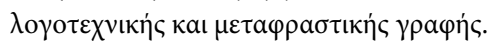

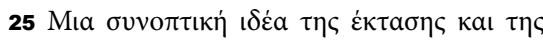

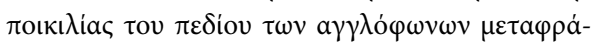

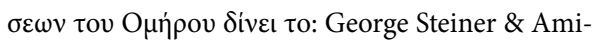
nadav Dykman (eds), Homer in English, London,

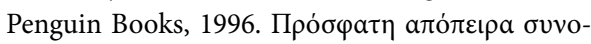

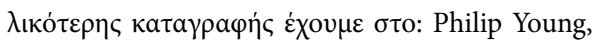
The Printed Homer: A 3000 Year Publishing and Translation History of the Iliad and the Odyssey, London, MacFarland Publishers, 2003.

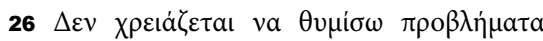

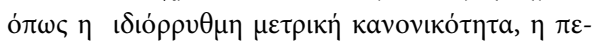

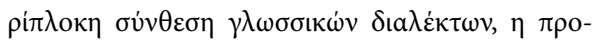

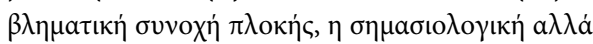

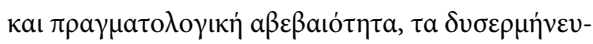

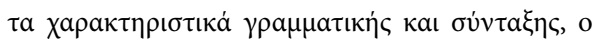

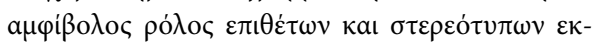
$\varphi \rho a ́ \sigma \varepsilon \omega v \dot{\eta} \lambda$ oเ $\pi \dot{\omega} v \varepsilon \pi \alpha v \alpha \lambda \dot{\eta} \psi \varepsilon \omega v$. 'О

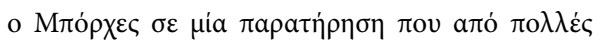

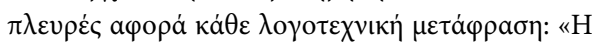

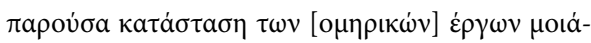

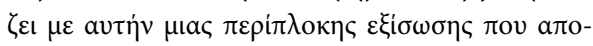

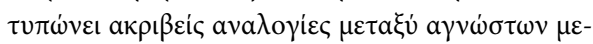

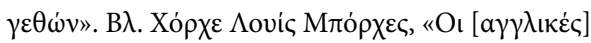

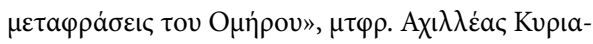

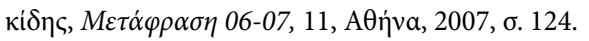

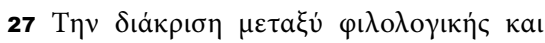
$\lambda о \gamma о \tau \varepsilon \chi \nu เ \kappa \eta ́ \varsigma ~ \mu \varepsilon \tau \dot{\alpha} \varphi \rho \alpha \sigma \eta \varsigma, \kappa \alpha \theta \dot{\omega} \varsigma \kappa \alpha \mathrm{l} \tau \alpha \sigma \chi \varepsilon \tau \mathrm{l}-$

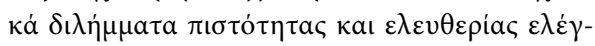

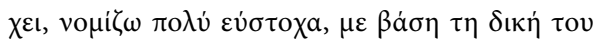

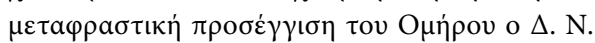

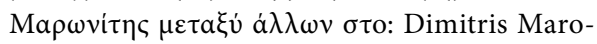
nitis, «Intralingual Translation: Genuine and False Dilemmas", in Alexandra Lianeri \& Vanda

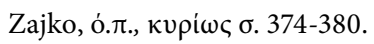

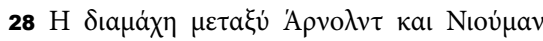

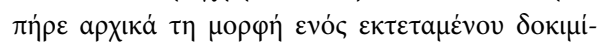

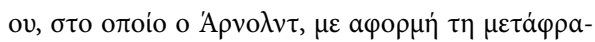

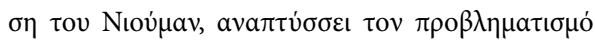

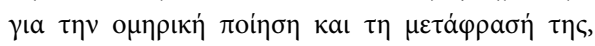

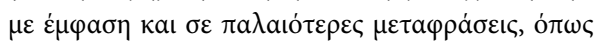

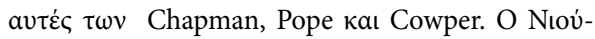

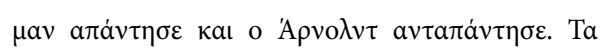

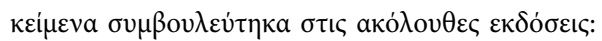
Mathhew Arnold, «On Translating Homer», in On the Classical Tradition, Ann Arbor, The University of Michigan Press, 1960 kal Francis Newman, Homeric Translation in Theory and Practice, London,

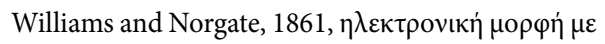

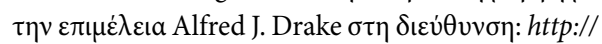
www.ajdrake.com/etexts/ (Electronic Version 1.0 /

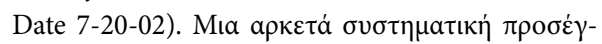

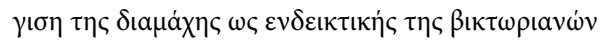

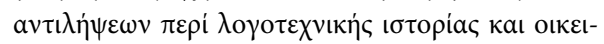

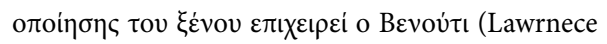
Venuti, ó. $\pi ., \sigma \sigma .118-145)$

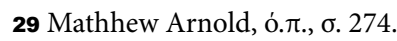

30 Francis Newman, ó. $\pi$., $\sigma .48$.

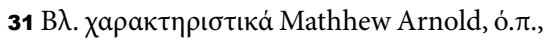
б. 136-137.

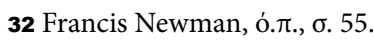

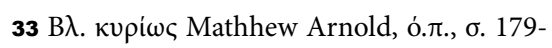
181.

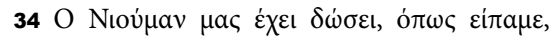

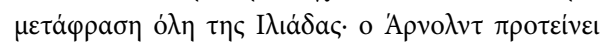

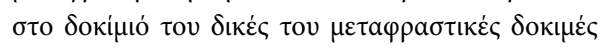

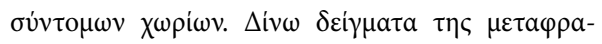

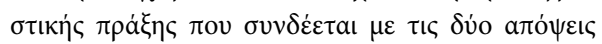

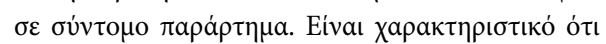

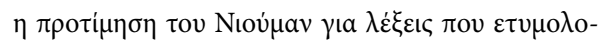

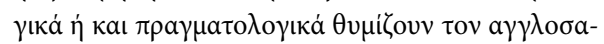

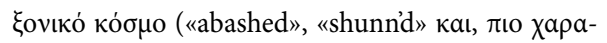

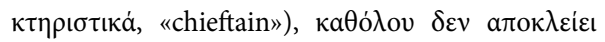
ava

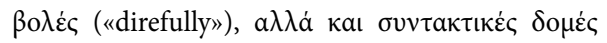

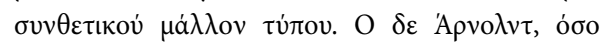

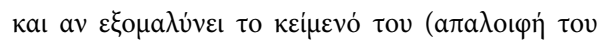

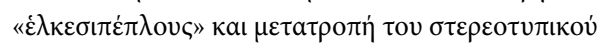

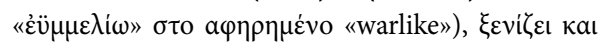

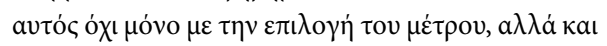

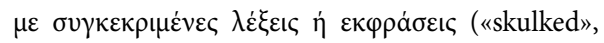

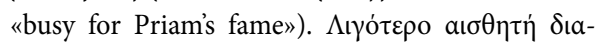


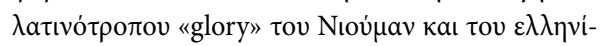

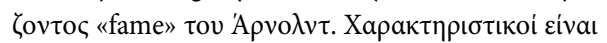

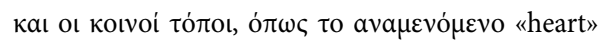

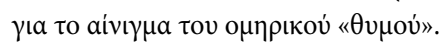




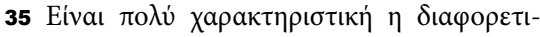

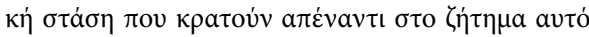

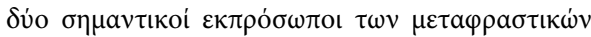

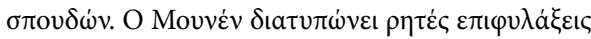

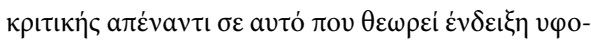

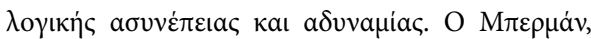

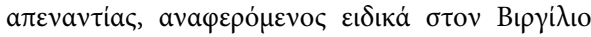

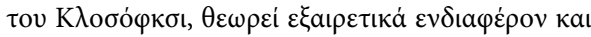

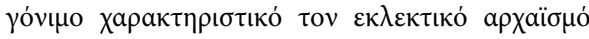

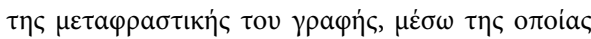

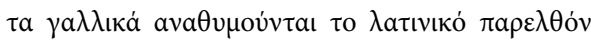

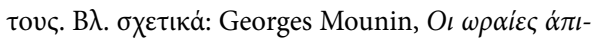

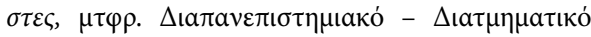

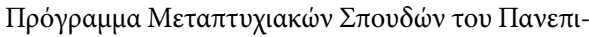

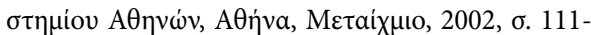

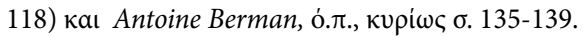

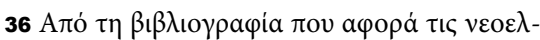

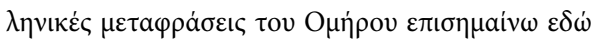

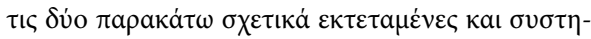

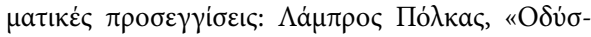

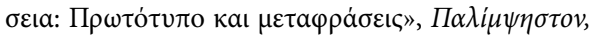

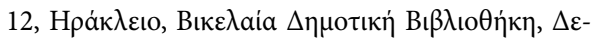

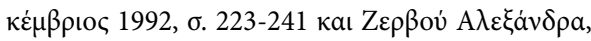

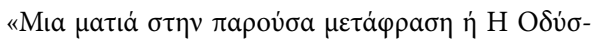

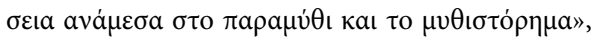

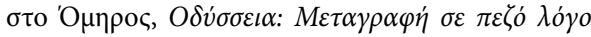

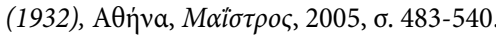

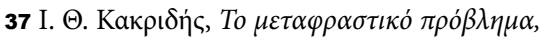

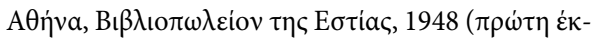

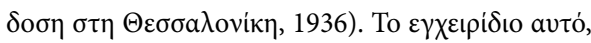

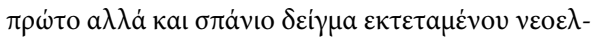

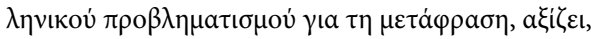

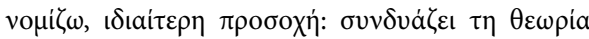

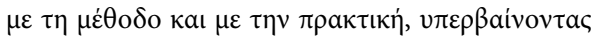

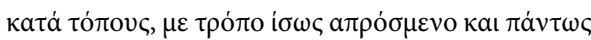

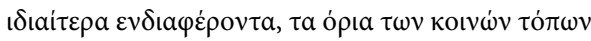

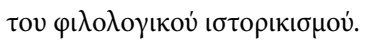

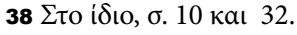

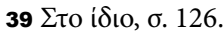

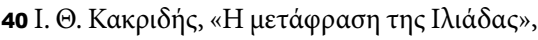

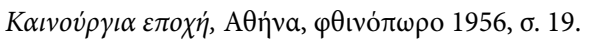

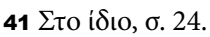

$42 \Sigma \tau$ i íıเo, $\sigma .34$.

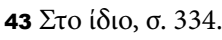

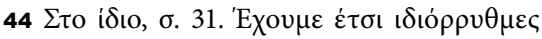

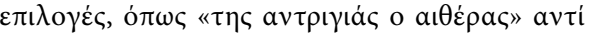

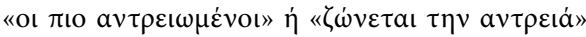

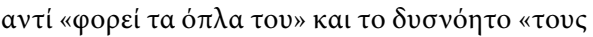

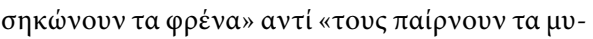

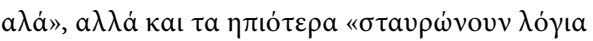

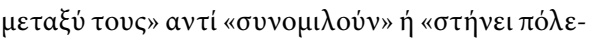
$\mu \mathrm{o»} \mathrm{\alpha \nu \tau i} \mathrm{« \alpha \rho \chi i \zeta \varepsilon l} \mathrm{\pi ó \lambda \varepsilon \mu o».}$

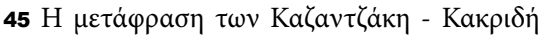

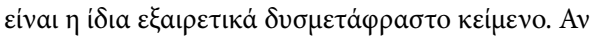

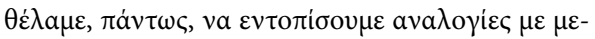

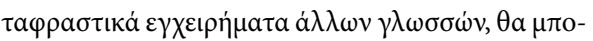

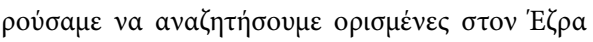

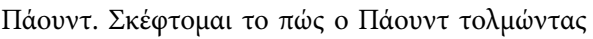

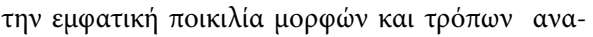

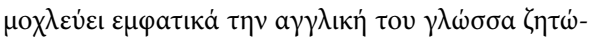

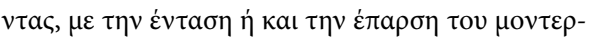

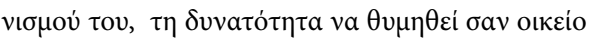

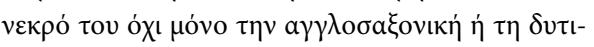

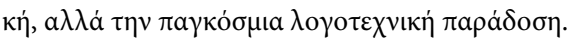

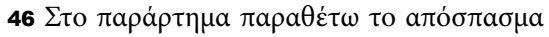

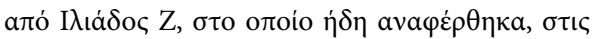

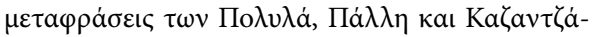

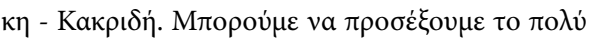

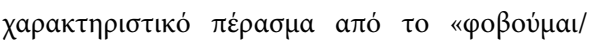

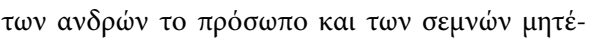

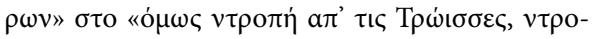

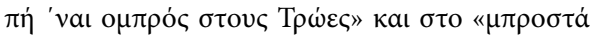

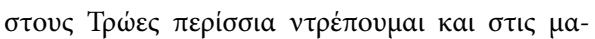

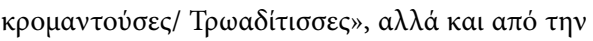

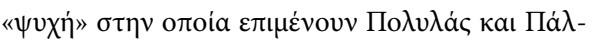

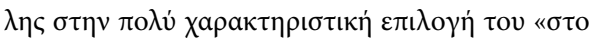

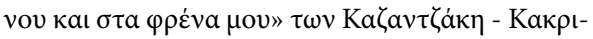

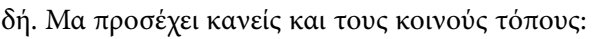

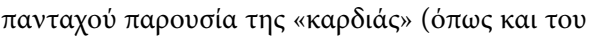

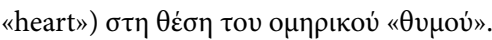

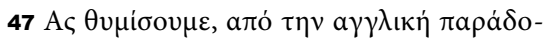
$\sigma \eta$, $\tau \iota \varsigma, \mu \varepsilon \tau \alpha \varphi \rho \alpha \dot{\sigma \varepsilon \iota \varsigma} \tau \omega v$ Richmond Lattimore

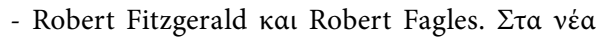
$\varepsilon \lambda \lambda \eta v i \kappa \alpha \dot{~} \delta \rho \alpha \sigma \tau \iota \kappa \eta ́ ~ \alpha \lambda \lambda \alpha \gamma \eta \dot{~ \tau o v ~ \tau o \pi i ́ o v ~ \varphi \varepsilon ́ \rho v \varepsilon ı ~}$

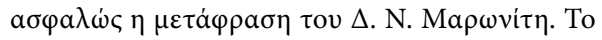

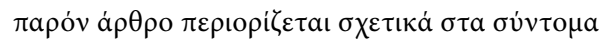

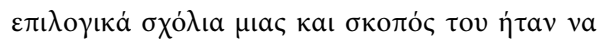

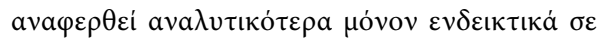
$\pi \varepsilon \rho ı \pi \tau \dot{\omega} \sigma \varepsilon ı \varsigma$ $\pi \alpha \lambda \alpha$ เó $\varepsilon \rho \omega \nu \mu \varepsilon \tau \alpha \varphi \varphi \rho \dot{\sigma \varepsilon} \omega \nu$. 


\section{RÉ S U M É}

GEORGES VARSOS: Écriture de traduction et mémoire: le cas des textes homériques.

Ce travail porte sur l'intérêt que la notion de mémoire présente pour l'analyse comparative de l' écriture traductrice, notamment dans le cas de la traduction des textes anciens. Sont discutées, dans un premier temps, certaines questions d'ordre théorique que pose Walter Benjamin aussi bien dans ses propres travaux que par l'intermédiaire d'une de ses références philosophiques les plus paradoxales, à savoir Henri Bergson. Nous tâchons ainsi de mettre en relief le rôle assumé par des procédés de mémoire dans la manière dont la traduction littéraire reconstruit l'idée même du passé et de sa survivance et entame des relations historiques qui dépassent les cadres des taxinomies et catégories afférentes données. Par ailleurs, l’approche proposée remet en question les polarités de la fidélité et de la liberté ou de la domestication et de la distanciation traductrices, en soulignant l'importance cruciale de l'incertitude et de l'instabilité sémantique et stylistique de l'écriture de la traduction. Dans sa deuxième partie, le travail discute la pertinence d'une telle approche pour l'étude du cas des traductions de la poésie homérique en différentes langues européennes modernes. On insiste, en particulier, sur le débat entre Matthew Arnold and Francis Newman pour ce qui est des traductions anglophones du XIXe siècle et sur celui de la traduction néohellénique de Kazantzakis - Kakridis. Cette dernière mettrait en évidence la spécificité du cas grec marqué par le double fait de la distance historique et de la parenté linguistique entre le passé de l'original et le présent de la traduction. 
